\title{
Model-independent bounds on the nonoscillatory explanations of the MiniBooNE excess
}

\author{
Vedran Brdar@, ${ }^{*}$ Oliver Fischer, ${ }^{\dagger}$ and Alexei Yu. Smirnov ${ }^{\ddagger}$ \\ Max-Planck-Institut für Kernphysik, 69117 Heidelberg, Germany
}

(Received 4 November 2020; accepted 17 March 2021; published 9 April 2021)

\begin{abstract}
We consider the nonoscillatory explanations of the low-energy excess of events detected by MiniBooNE. We present a systematic search for phenomenological scenarios based on new physics which can produce the excess. We define scenarios as series of transitions and processes which connect interactions of accelerated protons in target with single-shower events in the MiniBooNE detector. The key elements of the scenarios are production and decay of new light $\mathcal{O}(\mathrm{keV}-100 \mathrm{MeV}$ ) particles (fermions or/and bosons). We find about 20 scenarios with the minimal possible number of new particles and interaction points. In practice, they are all reduced to a few generic scenarios, and in this way we develop the effective theory of the MiniBooNE excess. We consider tests of the scenarios with near or close detectors in neutrino experiments T2K ND280, NO $\nu$ A, and MINER $\nu$ A as well as in NOMAD and PS191. The scenarios immediately connect the MiniBooNE excess and expected numbers of new physics events in these detectors. We compute the expected numbers of events as functions of the lifetimes and masses of new particles and confront them with the corresponding experimental bounds. We indicate scenarios that are excluded or strongly disfavored by one or several experiments. Given our general approach, this work can also be regarded as the effective theory of new physics at accelerator-based neutrino experiments, being relevant for future projects such as DUNE.
\end{abstract}

DOI: 10.1103/PhysRevD.103.075008

\section{INTRODUCTION}

The jury is still out on whether new physics effects are necessary for an explanation of the low-energy excess of the $e$-like events observed by MiniBooNE [1,2]. In this work, we assume that the answer to this question is affirmative. The popular explanation based on oscillations driven by mixing with a new eV-scale neutrino is very strongly disfavored, if not excluded. ${ }^{1}$ Not only the global neutrino oscillation fit [4] but also properties of the excess (energy and angular distributions) are behind the last statement.

In this connection, various nonoscillatory explanations of the excess were proposed. Most of them make use of possible misidentification of the MiniBooNE events which can be due to electrons, photons, or collinear $e^{+} e^{-}$as well

\footnotetext{
*vbrdar@mpi-hd.mpg.de

oliver.fischer@mpi-hd.mpg.de

\$smirnov@mpi-hd.mpg.de

${ }^{1}$ An alternative oscillation scenario was discussed in Ref. [3], where short-baseline oscillations are due to very strong medium potential generated by new resonance scattering of neutrinos on the local overdense relic neutrino background.
}

Published by the American Physical Society under the terms of the Creative Commons Attribution 4.0 International license. Further distribution of this work must maintain attribution to the author(s) and the published article's title, journal citation, and DOI. Funded by SCOAP ${ }^{3}$. as $\gamma \gamma$ pairs. The explanations are based on production and decay of new heavy neutrinos $N$ or/and bosons $B$ with masses $\mathcal{O}(\mathrm{keV}-100 \mathrm{MeV})$. They include

(i) the $N$ production in the MiniBooNE detector via the $\nu_{\mu}$ upscattering and then the radiative $N$ decay [5];

(ii) production of $N$ in the decay pipe via mixing in $\nu_{\mu}$ and further radiative decay along the beam line and, mainly, in the detector [6];

(iii) the $N$ production in the detector via $\nu_{\mu}$ upscattering and decay with the appearance of the $e^{+} e^{-}$pair. Two versions have been proposed: the three-body decay $N \rightarrow \nu e^{+} e^{-}$[7-9] and the two-body decay $N \rightarrow \nu B$ followed by the decay of an on-shell boson $B \rightarrow e^{+} e^{-}$. Here, $B$ can be a new gauge boson $Z^{\prime}$ [10] or a scalar $B=S$ [11-13]. In these models, $B$ has a decay length which is much smaller than the size of the detector, $\lambda_{B} \ll d^{\mathrm{MB}}$, so that the event looks like a local decay of $N$. There is an important kinematical difference from the three-body decay of $N$ [7], since here the invariant mass of the pair $e^{+} e^{-}$is determined by the mass of $B$, which is smaller than the mass of $N$.

(iv) The $N$ production via mixing in the decay pipe followed by the decay $N \rightarrow \nu_{e} \phi$ along the baseline with emission of $\nu_{e}$. The latter, in turn, produces an electron via the charged-current quasi-elastic (CCQE) scattering in the detector [14-16] (see also [17]). 
(v) Production of the light scalar $B$ in the $\nu_{\mu}$ upscattering: $\nu_{\mu} A \rightarrow N B A^{\prime}$, which then decays as $B \rightarrow e^{+} e^{-}$ [18]. (In the model [18], $B$ is produced via coupling with the gauge boson mediator of the upscattering process.) The new neutrino $N$ does not contribute to the MiniBooNE signal, in contrast to the previous mechanisms.

It should be mentioned that a number of explanations do not reproduce the Liquid Scintillator Neutrino Detector (LSND) excess in contrast to oscillations (see Table I).

Recent measurements of the bunch timing [2] do not show a deviation (shift or widening) of the time distribution of the MiniBooNE events from the one due to usual light neutrinos [2]. This essentially excludes mechanisms of decay of heavy neutrinos in the second item above and restricts parameters of the mechanism in the first item.

Do other possibilities of this type exist, or is everything already covered? In this connection, we perform a systematic search of all possible phenomenological scenarios that can explain the MiniBooNE excess. We identify the simplest scenarios with a minimal number of new particles and new interaction points. Clearly, an increase of these points would introduce additional smallness, since there are various restrictions on new interactions.

The goal of this paper is to perform model-independent tests of explanations of the MiniBooNE excess. For this, we introduce scenarios, that is, sets of transitions and processes which connect proton interactions on target with the appearance of single-shower events in MiniBooNE. To test the explanations, we use data from accelerator neutrino experiments with near or relatively close detectors. The scenarios allow us to directly connect numbers of events in these detectors with the MiniBooNE excess. Various model-dependent features cancel in this consideration. We describe these scenarios by a small number of parameters. Notice that the scenarios can be further (and, in some cases, even more strongly) restricted by other observations.

The paper is organized as follows. In Sec. II, we present a systematic search for the simplest phenomenological scenarios which explain the MiniBooNE excess. In relevant

TABLE I. Mapping of the proposed models that aim at explaining the MiniBooNE anomaly (references in the first column) onto scenarios introduced in this paper (second column). In the third column, check marks (Xs) indicate whether a given proposal can (cannot) fit the LSND data.

\begin{tabular}{lcc}
\hline \hline Model & Scenario & LSND \\
\hline$[6]$ & $M_{N} D_{\gamma}$ & $\boldsymbol{x}$ \\
{$[5]$} & $U_{N} D_{\gamma}$ & $\boldsymbol{x}$ \\
{$[7-9]$} & $U_{N} D_{e e}$ & $\boldsymbol{x}$ \\
{$[10-12]$} & $U_{N} D_{B} D_{e e}$ & $\boldsymbol{x}$ \\
{$[13]$} & $U_{N} D_{B} D_{e e}$ & $\mathcal{J}$ \\
{$[14-17]$} & $U_{N} D_{\nu} U_{e}$ & $\boldsymbol{J}$ \\
{$[18]$} & $U_{B} D_{e e}$ & $\boldsymbol{x}$ \\
\hline \hline
\end{tabular}

aspects, they are reduced to a few qualitatively different possibilities. In Sec. III, we present general formulas for the number of events in the detectors as functions of the parameters of the experimental setups and the parameters of the scenarios. The latter mainly include the lifetimes and masses of new particles. In Sec. IV, we present parameters of the employed experiments and derive experimental upper bounds on the number of events due to new physics. In Sec. V, we compute the expected number of events due to new physics in different scenarios and confront them with experimental bounds. Discussion and conclusions follow in Sec. VI.

\section{SCENARIOS FOR THE MINIBOONE EXCESS}

\section{A. General bounds on explanations of the excess}

MiniBooNE (MB) observed the excesses of $1 s h$ events of $560.6 \pm 119.6$ and $77.4 \pm 28.5$ in the neutrino and antineutrino mode (horn polarities), respectively [1]. The collected data correspond to $18.75 \times 10^{20}$ protons on target (POT) $\left(11.27 \times 10^{20} \mathrm{POT}\right)$ in neutrino (antineutrino) mode. We will use the sum of the $\nu$ and $\bar{\nu}$ excesses:

$$
N_{1 s h, \text { exp }}^{\mathrm{MB}}=638.0 \pm 132.8
$$

We assume that this excess is due to new physics rather than underestimated or missed background or oscillations related to existence of the eV-scale sterile neutrino.

The source of events is the $8 \mathrm{GeV}$ proton beam from the booster that hit the beryllium target, producing secondary particles. The 818-ton liquid scintillation detector observes via the Cherenkov radiation the single-shower $(1 s h)$ events:

$$
p+A[\text { target }] \rightarrow[X] \rightarrow 1 \text { sh events [detector }] .
$$

The recoil nucleon can produce scintillation, but this additional source of light was not considered in the MB reconstruction of events. ${ }^{2}$ The MiniBooNE detector is not capable to identify particle(s) which induce these EM showers.

The appearance of the $1 s h$ events is time correlated with the $p A$ collisions in the target. Therefore, it should be a mediator(s) system $X$ which connects the ends: the $p A$ interaction in the target and the EM shower in the detector. Furthermore, the arrival time distribution of events was found to be consistent with the arrival time of the usual neutrinos. We will not discuss the LSND result: The requirement of joint explanation imposes additional restrictions on scenarios.

\footnotetext{
${ }^{2}$ Being included in the analysis, the information on the recoil could help exclude various possibilities and distinguish between the decay and upscattering explanations.
} 
What is the "black box" $X$ in Eq. (2)? It can be production and propagation of new particles or some new dynamics related to known particles like Lorentz violation [19], nonstandard decoherence [20], etc. We will assume that (i) the mediator system is some new particle (or system of particles) $X_{s}$ that is produced in the source, (ii) $X_{s}$ evolves, in general, via a chain of processes: $X_{s} \rightarrow X_{\text {det }}$, and (iii) then $X_{\text {det }}$ interacts or decays in the detector producing the 1 sh events:

$$
p+A[\text { target }] \rightarrow X_{s}[\rightarrow] X_{\text {det }} \rightarrow 1 s h[\text { detector }] .
$$

There are certain observations that allow us to eliminate many possibilities and make the first step toward connecting "the ends":

(1) The proton beam energy $E \sim 8 \mathrm{GeV}$ restricts the mass scale of new particles to be at most around a few $\mathrm{GeV}$. Since charged particles at this mass scale are excluded, the new particles should be electrically neutral.

(2) The numbers of excess events as compared to the $\nu_{\mu^{-}}$ and $\nu_{e}$-CC events equal

$$
\frac{N_{1 s h}^{\mathrm{MB}}}{N_{\mu}^{\mathrm{MB}}} \simeq 10^{-2}, \quad \frac{N_{1 s h}^{\mathrm{MB}}}{N_{e}^{\mathrm{MB}}}=0.53 .
$$

Therefore, the processes which lead to the excess should not be very rare. In fact, the yield should be comparable to the yield of usual neutrinos unless we assume that $X$ has strong interaction.

(3) The excess is absent in the beam-dump run [21]: In this run, according to the number of POTs, about 30 events should be produced, but no excess was observed.

(4) The ratio of excesses in $\nu$ and $\bar{\nu}$ modes (horn polarities) corresponds to what is expected for usual neutrinos.

The implications of these results follow.

From the source side.-In general, $X_{s}$ can be produced

(i) on target in the $p A$ collisions immediately,

(ii) in decays (interactions) of known particles produced in the $p A$ collisions, such as $\pi, K$, and heavy mesons, and

(iii) by usual neutrinos $\nu_{\mu}$ in the detector or/and surrounding matter along the baseline.

The beam-dump mode results and the $\nu-\bar{\nu}$ results exclude the first possibility. The number of excess events in $\nu$ and $\bar{\nu}$ modes corresponds to what is expected for usual neutrinos, which implies the same differences of $X_{s}$ production and $X_{\text {det }}$ interaction as for neutrinos. Neutral particle decays as sources of $X_{s}$ are excluded, since they are not affected by the magnetic field and beam dump [22]. Thus, we arrive at the conclusion that $X_{s}$ should be produced in the charged $\pi$ and $K$ decays immediately or by the usual neutrinos from these decays.

Notice that, apart from three possibilities described above, one can consider production of $X_{s}$ in upscattering of muons from $\pi$ and $K$ decays in a shield and dirt.

From the detector side.-The 1 sh MiniBooNE events can be produced by $e, \gamma$, a collimated $e^{+} e^{-}$pair, and a collimated $\gamma \gamma$ pair, that is, by state $\xi$ :

$$
\xi=e, \gamma, e^{+} e^{-}, \gamma \gamma
$$

We will not consider more complicated systems, since their production will bring additional suppression. Fluxes of particles $\xi$ from the outside are suppressed by absorption in walls of the detector, rejection by the anticoincidence system, and fiducial volume cut. Furthermore, radial distribution of events shows that the excess increases toward the center [2]. Therefore, $X_{\text {det }}$ in Eq. (3) should be some neutral particle that enters MiniBooNE and produces $\xi$ in interaction or decay inside the detector.

The particle(s) $X_{\text {det }}$ as well as $X_{s}$ can be fermion $N$ or boson $B$, and the latter can be scalar or vector bosons. For definiteness, we will mainly explore spin- $1 / 2$ fermion $^{3}$ and boson cases: $X=N, B$.

If $X_{s}$ is a new heavy neutrino $X_{s}=N$, it can be produced via mixing in $\nu_{\mu}$. Therefore, the relevant channels of production are the same as for $\nu_{\mu}$ with substitution $\nu_{\mu} \rightarrow N$. If $X_{s}=B$, the decays are the same as the standard decay modes of $K$ and $\pi$ with additional $B$ emission (bremsstrahlung): $K \rightarrow \mu \nu B$ and $\pi \rightarrow \mu \nu B$, or standard modes in which one of pions is substituted by $B: K \rightarrow$ $\pi B$ and $K \rightarrow \pi \pi B$. Details of these decays, values of couplings, bounds, etc., are not important for our analysis.

The electromagnetic systems $\xi$ (5) can be produced in decays of $N$ or in $N$ interactions. Because of fermionic nature, the $N$ decays can proceed with emission of the usual neutrinos or a new neutral fermion $N^{\prime}$ :

$$
N \rightarrow \nu+\xi, \quad N \rightarrow N^{\prime}+\xi .
$$

The simplest possibilities include the radiative decay $(\xi=\gamma)$

$$
N \rightarrow \nu+\gamma,
$$

the three-body decay $\left(\xi=e^{+} e^{-}\right)$

$$
N \rightarrow \nu+e^{+}+e^{-}
$$

and decay via production of an on-shell boson (double decay):

\footnotetext{
${ }^{3}$ Spin-3/2 particles, like the gravitino, can also be considered.
} 


$$
N \rightarrow \nu+B, \quad B \rightarrow e^{+}+e^{-} \quad \text { or } \quad B \rightarrow \gamma+\gamma .
$$

Here, $B$ can be $\pi^{0}$ or some new scalar or vector boson.

Alternatively, $\xi$ can be produced in $N$ interactions with electrons or nucleons $(A)$ :

$$
N+e \rightarrow e+N^{\prime}, \quad N+A \rightarrow e+A^{\prime},
$$

where $N^{\prime}$ can coincide with the usual neutrinos $\nu_{\mu}$ or $\nu_{e}$.

In the case of a new boson, $X_{\mathrm{det}}=B$, the state $\xi$ can be produced in the two-body decays:

$$
B \rightarrow e^{+}+e^{-}, \quad B \rightarrow \gamma+\gamma, \quad B \rightarrow B^{\prime}+\gamma,
$$

or the three-body decay:

$$
B \rightarrow B^{\prime}+e^{+}+e^{-} .
$$

Also, $\xi$ can appear in $B$ interactions with nuclei and electrons:

$$
B+A \rightarrow A+e^{+}+e^{-}, \quad B+A \rightarrow A+\gamma, \quad B+e \rightarrow B+e .
$$

\section{B. Combinatorics of connections: Scenarios}

Let us consider all possible connections of the source and detector parts, i.e., transition $X_{s} \rightarrow X_{\text {det }}$. In the simplest case, $X_{s}$ and $X_{\text {det }}$ coincide: $X_{s}=X_{\text {det }}$. The next possibility is that $X_{\text {det }}$ is produced in decays of $X_{s}$ or in interactions of $X_{s}$ with the medium on the way to a detector or inside the detector. Several particles can be involved via a chain of processes connecting the ends: $X_{s} \rightarrow X_{1} \rightarrow X_{2} \ldots \rightarrow X_{\text {det }}$. At this point, we will employ criteria of minimality: The simplest links with the minimal number of chains or interaction points will be identified. Notice that, in general, any new vertex or additional new particle typically brings an additional suppression, and it is difficult to produce the required number of events in MiniBooNE.

Let us consider transitions with two and more interaction points which include production and decay of a new fermion $N$ or boson $B .^{4}$

Heavy neutrino $N$ can be produced

(i) in decays of usual mesons $\pi$ and $K$ in a decay pipe (for $N$, it is due to mixing with the usual neutrinos) -we call this element of the scenario $M$ (mixing) -or

\footnotetext{
${ }^{4}$ Notice that the simplest scenario would be with a single nonstandard interaction vertex, when $X_{s}=X_{\text {det }}=\nu_{\mu}$. Now, $\nu_{\mu}$, from standard $\pi$ and $K$ decays, produce electrons in the detector via the charged current nonstandard interaction (CC NSI) $\nu_{\mu}+$ $A \rightarrow e+A^{\prime}$ (this implies that $\nu_{\mu}$ is not orthogonal to $\nu_{e}$ ) or via neutral current (NC) NSI on electrons. Such a possibility is restricted very strongly.
}

(ii) by the $\nu_{\mu}$ interactions with matter outside the pipe, that is, by the $\nu_{\mu}$ upscattering $U_{N}$.

In the mixing case, the $N$ flux is formed in the decay pipe, while in the $U_{N}$ case, $N$ are produced outside the pipe.

In turn, $N$ can decay

(i) immediately into $\xi$ (we denote this process by $D_{\xi}$ );

(ii) into a state with $\nu_{e}$ and $D_{\nu}$, which then produces $\xi=e$ interacting in the detector $\left(U_{e}\right)$; and

(iii) into new neutral particles $N \rightarrow B$ which then decay into $\xi\left(D_{B} D_{\xi}\right)$.

Instead of decay, $N$ can upscatter on nucleons and electrons in a detector and outside the detector in dirt to produce $\xi\left(U_{\xi}\right)$. But this would involve another smallness due to additional nonstandard interaction. Indeed, the probability of $N$ interactions equals $P_{N}=\sigma_{N} n l$, where $\sigma_{N}$ is the cross section, $n$ is the number density of scatterers, and $l$ is the length of trajectory along which $N$ interacts. For new four-fermion interactions characterized by coupling $G_{N}$ and $\sigma_{N} \propto G_{N}^{2} E_{N}$, where $E_{N}$ is the energy of $N$, we obtain

$P_{N} \approx 5 \times 10^{-11}\left(\frac{l}{10 \mathrm{~m}}\right)\left(\frac{n}{3 n_{A}}\right)\left(\frac{E_{N}}{1 \mathrm{GeV}}\right)\left(\frac{G_{N}}{G_{F}}\right)^{2}$,

where $n_{A}$ is the Avogadro number. Let us compare this probability with the probability of $N$ decay. If $N$ is produced at the distance $l$ from a detector and the size of a detector is $d$, then the probability of its decay in the detector equals

$$
P_{\mathrm{dec}}=e^{-l / \lambda_{N}}\left(1-e^{-d / \lambda_{N}}\right) .
$$

Here, $\lambda_{N}$ is the decay length of $N$ :

$$
\lambda_{N}\left(E_{N}, m_{N}\right)=\frac{E_{N}}{m_{N}} c \tau_{N}^{0},
$$

where $c$ is the velocity of light, $\tau_{N}^{0}$ is the lifetime of $N$ in the rest frame, and $m_{N}$ is the mass of $N$.

For fixed $l$ and $d$, the maximum of $P_{\mathrm{dec}}$ is achieved at

$$
\lambda_{N}=d[\log (1+d / l)]^{-1} \approx l,
$$

where the second equality is for $d \ll l$. The probability at $\lambda_{N}=l$ and typical values of $d$ and $l$ equals

$$
P_{\text {decay }}^{\max }=\frac{d}{e l} \sim 10^{-2}
$$

( $e \approx 2.7$ ). Therefore, the $N$ decay can be substituted by upscattering of $N$, if $P_{N}>10^{-2}$, which implies, according to Eq. (6), that $G_{N}>10^{4} G_{F}$. The latter is difficult to realize.

Connecting two $N$-production mechanisms (mixing and upscattering) and three decay possibilities listed above, we 
arrive at the following six scenarios for $X=N$. The number of possibilities multiplies due to various $\xi$ (5).

(i) $M_{N} D_{\xi}$, mixing-decay scenario. $-N$ is produced in the $K$ and $\pi$ decay via mixing in $\nu_{\mu}$, and it decays as $N \rightarrow N^{\prime}+\xi$. Here, $\xi$ is any state in Eq. (5) except the electron, and $N^{\prime}$ can be a standard neutrino $\nu$. Only decays inside a detector give an observable signal.

(ii) $M_{N} D_{\nu} U_{e}$, mixing-decay into $\nu_{e}$ scenario. $-N$ produced via mixing decays with emission of $\nu_{e}$ : $N \rightarrow \nu_{e}+B$. Then $\nu_{e}$ upscatters in the detector, producing an electron.

(iii) $M_{N} D_{B} D_{\xi}$, mixing-double-decay scenario. $-N$ produced via mixing decays invisibly into another new particle $B$, which, in turn, decays into (or with emission of) $\xi$.

(iv) $U_{N} D_{\xi}$, upscattering-decay scenario. $-N$ is produced in the $\nu_{\mu}$ interactions with particles of medium between a source and a detector as well as inside the detector. Then $N$ decay in the detector produces $\xi$. If interactions of $N$ with medium can be neglected, the $N$ flux will be accumulated along the way to a detector.

(v) $U_{N} D_{\nu} U_{e}$, upscattering-decay into $\nu_{e}$ scenario. $-N$ produced by the $\nu_{\mu}$ upscattering decays with emission of $\nu_{e}$, which then scatters in the detector via $\mathrm{CCQE}$, producing the $e$ shower.

(vi) $U_{N} D_{B} D_{\xi}$, upscattering-double-decay scenario. $-N$ produced by the $\nu_{\mu}$ upscattering undergoes double decay: $N \rightarrow B \rightarrow \xi$.

Scenarios (i), (ii), (iv), and (v) contain two vertices with new particles; scenarios (iii) and (vi) are of higher (third) order in new physics interactions.

Two more scenarios can be identified in which $\xi$ state is produced by upscattering of $N$. They have additional suppression in comparison to $\xi$ production in decays. The first scenario is $M_{N} U_{\xi}$, i.e., the mixing- $N$ upscattering. Here, $N$ produced via mixing in $\nu_{\mu}$ upscatters in a detector with production of an electron: $N+A \rightarrow e+A^{\prime}$. This implies lepton number violation, since $N$ is mixed in $\nu_{\mu}$ but produces $e$ in interactions. The second scenario is $U_{N} U_{\xi}$, which is double upscattering. $N$ is produced in upscattering of $\nu_{\mu}$ and then upscatters with production of $\xi(e)$.

The six scenarios described above are not completely independent from the geometrical point of view and even coincide in certain limits of values of parameters. Thus, for a short lifetime of $B$ we have

$$
U_{N} D_{\xi} \approx U_{N} D_{B} D_{\xi},
$$

with the only difference that in the double-decay case the invariant mass of particles in the final state is fixed by the mass of $N$.
For $X=B$, we have similar mechanisms of production and decay. As far as propagation features are concerned, the scenarios with $B$ coincide with scenarios for $N$ but differ from the model-building side. Also in this case, instead of mixing in $\nu_{\mu}, B$ are produced in $\pi$ and $K$ decays, and, therefore, $M$ should be interpreted as $B$ production in the meson decays. For bosons, we have the following scenarios:

(i) $M_{B} D_{\xi}$ - - production of $B$ in a decay pipe in meson decays and further decay $B \rightarrow \xi, B \rightarrow B^{\prime} \xi$;

(ii) $M_{B} D_{\nu} D_{e} \cdot-B$ decays with emission of $\nu_{e}, B \rightarrow \nu_{e} \bar{\nu}_{e}$ or $B \rightarrow \nu_{e} N^{\prime}$; and

(iii) $M_{B} D_{B^{\prime}} D_{\xi}$.- double decay, which is a nonminimal and complicated version of (i).

Three other mechanisms differ from (i)-(iii) by the $B$ production mechanism; namely, instead of decays in a pipe, $B$ is produced via $\nu_{\mu}$ upscattering in a detector and the surrounding medium. These three scenarios include

(iv) $U_{B} D_{\xi}$ - - with $B$ decays as in (i) (see Ref. [18]);

(v) $U_{B} D_{\nu} D_{e} \cdot-B$ decay into $\nu_{e}$, which, in turn, produces $e$ in $\mathrm{CCQE}$ in a detector; and

(vi) $U_{B} D_{B^{\prime}} D_{\xi}$. - double decay, which is nonminimal version of (iv).

Throughout the paper, we focus on scenarios with $X=N$.

In Table I, we associate the proposed nonoscillatory models for the MiniBooNE anomaly to the aforementioned scenarios. There, we also indicate whether a given proposal can also reasonably well explain the LSND anomaly (third column).

\section{Bounds on parameters of scenarios from timing}

The key parameters of the scenarios are masses and lifetimes of new particles. Therefore, the bounds from timing of the MB events are crucial for our consideration. The bounds differ for scenarios with $N$ production in a decay pipe via mixing and in a detector via upscattering. In the first case, $N$ propagates from a production point in a pipe to a detector; i.e., the distance equals the baseline, $l$. A delay of the events produced by $N$ with respect to the signal from usual neutrinos equals

$$
\Delta t=\frac{l}{c}\left[\frac{1}{\sqrt{1-\left(m_{N} / E_{N}\right)^{2}}}-1\right] \approx \frac{l}{c} \frac{m_{N}^{2}}{2 E_{N}^{2}},
$$

and the last equality in Eq. (12) is for $m_{N} / E_{N} \ll 1$. Numerically, we have

$$
\Delta t=8 \mathrm{~ns}\left(\frac{l}{500 \mathrm{~m}}\right)\left(\frac{m_{N}}{0.1 \mathrm{GeV}}\right)^{2}\left(\frac{1 \mathrm{GeV}}{E_{N}}\right)^{2} .
$$

Using the typical excess energy $E_{N}=0.3 \mathrm{GeV}$ and $\Delta t=1 \mathrm{~ns}$, we find from Eq. (13) the upper bound on the mass: $m_{N}<10 \mathrm{MeV}$. In the case of $N$ and $B$ decays, this bound leads to a very forward excess of events in 
MiniBooNE which contradicts the data. Indeed, the observed angular spectrum of the MiniBooNE excess requires $m_{N}$ to be above $200 \mathrm{MeV}$ [22]. Such a possibility can still be considered if there is a two-component interpretation of the angular distribution of the excess which, in fact, is favored by recent data. One component, e.g., due to underestimated background, is nearly isotropic and another one due to new physics contribution peaks in the forward direction. Keeping this in mind, we will consider such scenarios.

Another possibility is that $N$ and $B$ are produced via the $\nu_{\mu}$ (or another light particle) upscattering. In the upscattering case, the typical decay length is smaller than the detector size: $\lambda_{N}<d$. Therefore, we should take $\lambda_{N}$ as a conservative estimate of the distance of $N$ decay. Substituting $l$ by $\lambda_{N}=c \tau^{0} E_{N} / m_{N}$ in the expression (12), we can write the upper bound on the lifetime of $N$ which ensures a delay smaller than a given $\Delta t$ :

$$
c \tau^{0}<c \Delta t \frac{m_{N}}{E_{N}}\left[\frac{1}{\sqrt{1-\left(m_{N} / E_{N}\right)^{2}}}-1\right]^{-1} .
$$

For $m_{N} / E_{N} \ll 1$, this gives

$$
c \tau^{0}<2 c \Delta t \frac{E_{N}}{m_{N}}
$$

Taking $\Delta t=1 \mathrm{~ns}$ and $E_{N}=0.8 \mathrm{GeV}$, we obtain the following upper bounds on $c \tau^{0}$ for values $m_{N}=$ $(0.15,0.25,0.35) \mathrm{GeV}$, respectively:

$$
c \tau^{0}<(3.2,1.92,1.37) \mathrm{m} \text {. }
$$

$N$ production via $\nu_{\mu}$ upscattering usually implies $N$ mixing in $\nu_{\mu}$. Therefore, in general, one has to sum the contributions from $N$ produced via the mixing and upscattering mechanisms. However, these two mechanisms are effectively operative in different ranges of $c \tau^{0}$. In the upscattering case, $N$ should decay within the detector volume $\left(c \tau^{0} \leq 1 \mathrm{~m}\right)$ unless it decays into another new particle $B$, while in the case of $N$ production in a decay pipe via mixing, $N$ should reach the detector, i.e., survive about several hundred meters, implying that $c \tau^{0} \gtrsim 100 \mathrm{~m}$. Therefore, for a given value of $c \tau^{0}$, only one mechanism dominates.

\section{Signature factors and efficiencies}

A detector $i$ observes events of various types $s^{i}$, which depend on features of the detector. We will call $s^{i}$ signatures. In particular, MiniBooNE observes one- and two-shower events, while ND T2K with better particle ID can observe $\gamma$ showers, $e$ showers (tracks), and two-shower events:

$$
s^{\mathrm{MB}}=\{1 s h, 2 s h\}, \quad s^{\mathrm{ND}}=\{\gamma-s h, e-s h, 2 s h\} .
$$

Because of misidentification, the observed events do not correspond uniquely to certain original states $\xi$. To quantify this, we introduce the signature factors $f_{\xi-s^{i}}^{i}$, which give the fraction of cases in which a given state $\xi$ shows up as an $s^{i}$ event in the $i$ detector. Equivalently, $f_{\xi-s^{i}}^{i}$ can be considered as the probability that a state $\xi$ will show up as an $s^{i}$ event.

$f_{\xi-s^{i}}^{i}$ depends on the parameters of the state $\xi$-energies of particles and masses-as well as on properties of detectors. For MiniBooNE, a single electron will be detected as a $1 s h$ event, namely, $f_{e-1 s h}^{\mathrm{MB}}=1$. Similarly, for $\gamma, f_{\gamma-1 s h}^{\mathrm{MB}}=1$. Also, an $e^{+} e^{-}$state can show up as a oneshower event, but $f_{e e-1 s h}^{\mathrm{MB}}<1$ and the fraction depends on the kinematical variables of $e^{+}$and $e^{-}$.

The numbers of events depend also on experimental reconstruction efficiency for a given signature $\epsilon_{s}^{i}\left(E_{N}, m_{N}\right)$. It is an empirical function which depends on properties of the signature, such as energies and angles. For simplicity, we take it to be a constant value for a given experiment and signature.

We can introduce the signature factor in a different way (taking one step back), considering the final process (decay or scattering) in which the state $\xi$ is produced. Then one can introduce $f_{s^{i}}$ as a fraction of $N$ decays or $\nu$ scatterings in which the $s^{i}$ event is produced.

\section{NUMBERS OF NEW PHYSICS EVENTS IN THE GENERIC SCENARIOS}

\section{A. General expression for number of events}

For the scenarios described in Sec. II, we will compute the number of expected events of type $s^{i}$ in $i$ detector $N_{s, \text { exp }}^{i}$, in the following way:

$$
N_{\xi, \exp }^{i}=N_{1 s h, \exp }^{\mathrm{MB}} \frac{N_{\xi-s^{i}}^{i}}{N_{1 s h}^{\mathrm{MB}}},
$$

where $N_{1 s h \text {,exp }}^{\mathrm{MB}}$ is given in Eq. (1) and $N_{\xi-s^{i}}^{i}$ and $N_{\xi-1 s h}^{\mathrm{MB}}$ are the theoretical numbers of events in a detector $i$ and MiniBooNE correspondingly. That is, we normalize the numbers of events of type $\xi-s^{i}$ in a given detector $i$ to the MB excess of $1 s h$ events, $N_{1 s h}^{\mathrm{MB}}$. In this way, we ensure that a given scenario explains the MB excess. Furthermore, various factors cancel in the ratio of predictions such as mixing parameter, coupling constants, normalization of cross sections, etc.

The signal in $i$ detector predicted in terms of the MiniBooNE excess (18) is determined by the difference (ratio) of theoretical values of signals in the $i$ and MiniBooNE detectors. (Recall that we are considering experiments with qualitatively similar setups.) In what follows, we will derive general expressions for the numbers 
of events. Apart from the external parameters such as numbers of POT, $\epsilon$, and detector mass $M$, the difference stems from geometry - values of the length of decay pipe $l_{p}^{i}$, the distance between the end of the pipe and the detector $b^{i}$ so that $l^{i}=l_{p}^{i}+b^{i}$ is the total baseline, the effective length of a detector $d^{i}$, the energy spectra, and masses of particles involved, in particular, $m_{N}$ and $m_{B}$. The difference depends on characteristics of the detectors and, first of all, particle ID, efficiencies of event selection, etc., which are encoded in the signature factors. Other characteristics cancel.

For simplicity, superscripts $i$ that indicate the experiment or detector will be omitted. We will recover them when needed.

Scenarios for MiniBooNE excess are the chains of interactions and propagations of new as well as standard model particles. The interactions include upscattering and decays. In each interaction, one leading particle, $Y_{k}$, is absorbed and another one, $Y_{k+1}$, is produced, which eventually gives an observed signal $\xi$ in a detector. We assume that the leading particles move along the line which connects the source and detector, thus neglecting all the scattering and emission angles but the angles in the detector. The latter will be included into significance factors and efficiencies. At the same time, we will take into account the change of energy of the leading particle in all interactions. In a given interaction vertex $k$ with coordinate $x_{k}$, a leading particle with energy $E_{k}$ is absorbed and a leading particle with energy $E_{k+1}$ is emitted.

The general expression for the number of events can be written as a product of several factors $I_{k}$ associated to vertices $k$ of interactions. The initial flux is the flux of $\pi$ and $K$ mesons produced at a target $d \phi_{\pi}^{0}\left(E_{\pi}\right) / d E_{\pi}$ and $d \phi_{K}^{0}\left(E_{\pi}\right) / d E_{K}$. So, the first vertex is $\pi$ (or $K$ ) decay in a decay pipe: $I_{1}=D_{1}$. There are two possibilities:

(1) New particles $N$ or $B$ are produced in these decays.

(2) $\nu_{\mu}$ is produced and as the initial state we can consider the $\nu_{\mu}$ flux at the exit of the decay pipe $d \phi_{\nu}^{0}\left(E_{\pi}\right) / d E_{\nu}$. Since $\nu_{\mu}$ is stable, the first vertex should be upscattering: $I_{1}=U_{1}$.

In the 1D approximation (straight propagation of the leading particles), the flux integrated over time should be multiplied by the area of a detector $A$. For a vertex with decay, the following factors are associated:

$D_{k}\left(E_{k}\right)=\int d E_{k} \frac{d \Gamma_{k}\left(E_{k}, E_{k+1}\right)}{\Gamma_{k}^{\text {tot }} d E_{k+1}} \int \frac{d x}{\lambda_{k}} S_{k}\left(E_{k}, x_{k}-x_{k-1}\right)$.

Here,

$$
S_{k}\left(E_{k}, x_{k}-x_{k-1}\right) \equiv e^{-\left(x_{k}-x_{k-1}\right) / \lambda_{k}} \quad\left(\lambda_{k} \equiv \frac{E_{k}}{m_{k}} c \tau_{k}^{0}\right)
$$

is the survival probability: Since particle $Y_{k}$ (which enters vertex $k$ ) decays, it should survive between $x_{k}$ and the production point $x_{k-1}$. Notice that we cannot perform integration over $E_{k+1}$ in Eq. (19), since other factors in the product of $I_{i}$ on the rhs from a given $I_{k}$ can depend on $E_{k+1}$.

For a vertex with upscattering of stable particle $Y_{k}$, the factor reads as

$$
U_{k}\left(E_{k}\right)=\int d E_{k} \frac{d \sigma_{k}\left(E_{k}, E_{k+1}\right)}{d E_{k+1}} \int d x_{k} n_{k}\left(x_{k}\right),
$$

where $n_{k}(x)$ is the density of a layer in which $Y_{k}$ interacts. In the case of constant density, the spatial integral can be written as

$$
n_{k} l_{k} \int \frac{d x_{k}}{l_{k}},
$$

where we introduced $l_{k}$, the length of layer of the $k$ particle production, to make integrals dimensionless.

If the upscattered particle is unstable, a survival probability should be added under spatial integral in Eq. (21):

$$
\begin{aligned}
U_{k}^{\prime}\left(E_{k}\right)= & \int d E_{k} \frac{d \sigma_{k}\left(E_{k}, E_{k+1}\right)}{d E_{k+1}} \\
& \times \int d x_{k} n_{k}\left(x_{k}\right) S_{k}\left(E_{k}, x_{k}-x_{k-1}\right) .
\end{aligned}
$$

Thus, the general expression for the number of events in a scenario with $n$ vertices can be written as

$$
\begin{aligned}
N_{\xi-s}= & A \int d E_{\pi} \frac{d \phi_{\pi}^{0}\left(E_{\pi}\right)}{d E_{\pi}} \\
& \times \prod_{k=1}^{n-1} I_{k}\left(E_{k}\right) \times I_{n}\left(E_{n}\right) f_{\xi-s}\left(E_{\xi}\right) \epsilon,
\end{aligned}
$$

where $I_{k}=\left\{D_{k}, U_{k}, U_{k}^{\prime}\right\}$ are introduced in Eqs. (19), (21), and (22). This expression can be factorized into the part that depends on kinematic variables (energies) and the propagation part which depends on the coordinates. In particular, the propagation or decay part equals

$$
\begin{aligned}
P_{\mathrm{dec}}= & \Pi_{i} \int \frac{d x_{i}}{l_{i}}\left[S_{i}\left(E_{i}, x_{i}-x_{i-1}\right)\right]{ }^{g_{i}} \Pi_{j} \\
& \times \int \frac{d x_{j}}{\lambda_{j}} S_{j}\left(E_{j}, x_{j}-x_{j-1}\right) .
\end{aligned}
$$

Here, the first product of integrals over $i$ corresponds to upscattering vertices with $g=0$ for stable and $g=1$ for unstable upscattered particle $i$. The second product over $j$ corresponds to vertices with decays. In this expression, the order and limits of integrations depend on the specific scenario. 


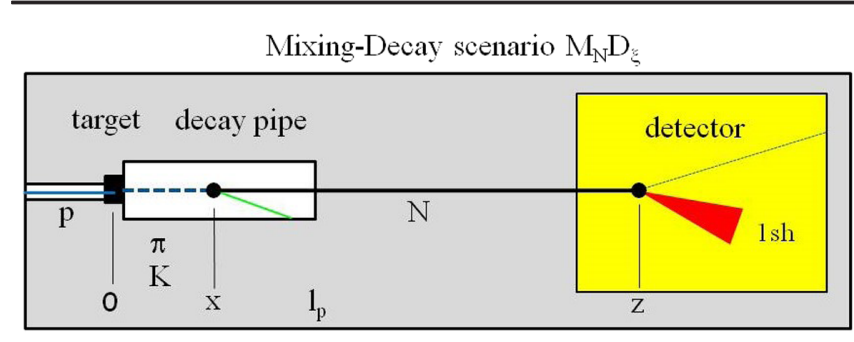

FIG. 1. Schematic depiction of the mixing-decay scenario. Black blobs show the interaction points, the red triangle denotes the EM shower, and $l_{p}$ is the length of the decay pipe.

Spins of the propagating (leading) particles are not important for general expression (23). They, however, are important for characteristics of interactions, decay rates, and cross sections.

\section{B. Mixing-decay, $M_{N} D_{\xi}$ scenario}

Recall that, in this scenario (schematically shown in Fig. 1), the heavy neutrinos $N$ are produced in the $\pi$ and $K$ decays via mixing in $\nu_{\mu}$ in a decay pipe. Then $N$ decays $(N \rightarrow \xi+\nu)$ along the baseline, from the production point in a pipe to a detector. Mostly, $N$ decays in a detector that produces the observable events. This mechanism gains with respect to upscattering mechanisms, since no interactions with matter in a detector is needed. But it loses because $N$ decays everywhere. (One expects lateral phenomena: some signal from $N$ decay outside a detector.) As we discussed, the optimal decay length, which maximizes the signal, is comparable to the baseline $\lambda \sim l$.

Because of the arrival time restrictions, $m_{N}<10 \mathrm{MeV}$ (see Sec. II C), $N$ is mainly produced in $\pi$ decays. Therefore, the initial flux is the $\pi$ flux at the target $d \phi_{\pi}^{0}\left(E_{\pi}\right) / d E_{\pi}$. This is the two-vertices scenario with decays in both vertices. Then, according to general formulas (23), the number of events in the detector is given by

$$
\begin{aligned}
N_{\xi-s}= & \epsilon A \int d E_{N} f_{\xi-s}\left(E_{N}\right) \\
& \times \int d E_{\pi} \frac{\phi_{\pi}^{0}\left(E_{\pi}\right)}{d E_{\pi}} \frac{d \Gamma_{\pi N}\left(E_{\pi}, E_{N}\right)}{\Gamma_{\pi}^{\mathrm{tot}} d E_{N}} P_{\mathrm{dec}}\left(E_{\pi}, E_{N}\right),
\end{aligned}
$$

where the mixing parameter $\left|U_{\mu 4}\right|^{2}$ is included in the decay rate $d \Gamma_{\pi N} / d E_{N}$. The decay factor (24) equals the integrals

$$
P_{\mathrm{dec}}=\int_{0}^{l_{p}} \frac{d x}{\lambda_{\pi}} S_{\pi}(x) \int_{l_{p}+b}^{l_{p}+b+d} \frac{d z}{\lambda_{N}} S_{N}(z-x)
$$

with the limits of integrations immediately seen in Fig. 1. In Eq. (26),

$$
S_{\pi}(x)=e^{-x / \lambda_{\pi}}, \quad S_{N}(z-x)=e^{-(z-x) / \lambda_{N}} .
$$

Explicit computation gives

$$
\begin{aligned}
P_{\mathrm{dec}}\left(E_{\pi}, E_{N}\right)= & e^{-b / \lambda_{N}}\left(1-e^{-d / \lambda_{N}}\right) \\
& \times\left(1-\frac{\lambda_{\pi}}{\lambda_{N}}\right)^{-1}\left[e^{-l_{p} / \lambda_{N}}-e^{-l_{p} / \lambda_{\pi}}\right] .
\end{aligned}
$$

Since $\lambda_{\pi} \ll \lambda_{N}$ and $\lambda_{\pi}<l_{p}$, the dependence of $P_{\mathrm{dec}}$ on $E_{\pi}$ is weak and $P_{\text {dec }}$ can be moved out of integration over $E_{\pi}$, with $E_{\pi}$ substituted by an effective pion energy $\bar{E}_{\pi}$. Then, by introducing the $N$ flux at the target, which would be in the case of stable $N$,

$$
\frac{\phi_{N}^{0}\left(E_{N}\right)}{d E_{N}} \equiv \int d E_{\pi} \frac{d \phi_{\pi}^{0}\left(E_{\pi}\right)}{d E_{\pi}} \frac{d \Gamma_{\pi N}\left(E_{\pi}, E_{N}\right)}{\Gamma_{\pi}^{\mathrm{tot}} d E_{N}}
$$

Eq. (25) can be reduced to

$$
N_{\xi-s}=\epsilon A \int d E_{N} \frac{d \phi_{N}^{0}\left(E_{N}\right)}{d E_{N}} f_{\xi-s}\left(E_{N}\right) P_{\mathrm{dec}}\left(\bar{\lambda}_{\pi}\right) .
$$

Here, $\bar{\lambda}_{\pi}=c \tau_{\pi}^{0} \bar{E}_{\pi} / m_{\pi}$. If also $d \ll \lambda_{N}$, the probability of decay in a detector is much smaller than 1 and the decay factor becomes

$$
P_{\mathrm{dec}} \approx \frac{d}{\lambda_{N}} e^{-l / \lambda_{N}}
$$

Qualitatively, the dependence of the predicted numbers of events (25) on $c \tau_{0}$ can be understood considering the ratio of the decay factors (30) for a given experiment $i$ and MiniBooNE taken at certain effective energies in experiments, $E^{i}$ and $E^{\mathrm{MB}}$ :

$$
r_{d} \equiv \frac{P_{\mathrm{dec}}^{i}}{P_{\mathrm{dec}}^{\mathrm{MB}}}=\left(\frac{d^{i}}{d^{\mathrm{MB}}}\right)\left(\frac{E_{N}^{\mathrm{MB}}}{E_{N}^{i}}\right) e^{\left(L^{\mathrm{MB}}-L^{i}\right) / c \tau^{0}},
$$

where

$$
L^{i} \equiv l^{i} \frac{m_{N}}{E_{N}^{i}} .
$$

According to Eq. (32), the dependence of $N_{s}^{i}$ on $c \tau^{0}$ is determined by baseline lengths rather than sizes of detectors. Among all the detectors we consider, $l$ is the longest and $E_{N}$ is the smallest for MiniBooNE; therefore, $L^{\mathrm{MB}}>L^{i}$. Numerically,

$$
L^{\mathrm{MB}}=6.7 \mathrm{~m}\left(\frac{m_{N}}{10 \mathrm{MeV}}\right) .
$$

For $c \tau^{0} \gg\left(L^{\mathrm{MB}}-L^{i}\right)$, the ratio $r_{d}$, and consequently $N_{\xi-s}^{i}$, does not depend on $c \tau^{0}$ as well as $m_{N}$. In this limit, decays of $N$ before the detector can be neglected. With a decrease of $c \tau^{0}$, first the MiniBooNE detection is affected by the $N$ decays and then the $i$ detector is. As a result, at 


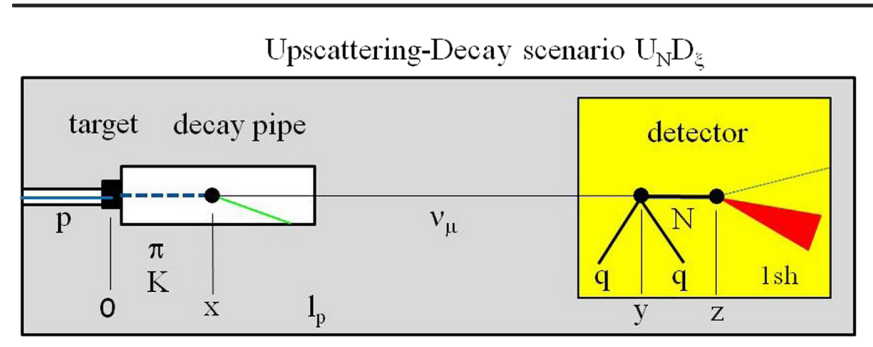

FIG. 2. The same as in Fig. 1 but for the upscattering-decay scenario.

$$
c \tau^{0}<c \tau_{\mathrm{up}}^{0} \equiv L^{\mathrm{MB}}-L^{i}=m_{N}\left(\frac{l^{\mathrm{MB}}}{E_{N}^{\mathrm{MB}}}-\frac{l^{i}}{E_{N}^{i}}\right)
$$

the ratio turns up and shows exponential growth (in agreement with figures in Sec. V). With an increase of $m_{N}$, the upturn shifts to larger $c \tau_{0}$. The dependence of the number of events on $m_{N}$ is determined in addition by the $m_{N}$ dependence of the $N$ fluxes, cross sections, and signature factors.

In the asymptotics $c \tau^{0} \gg \Delta L$, the theoretical number of events can be estimated using Eqs. (29) and (30) as

$$
N_{\xi-s}=\epsilon A d \frac{m_{N}}{c \tau^{0}} \int d E_{N} \frac{1}{E_{N}} f_{\xi-s}\left(E_{N}\right) \frac{d \phi_{N}^{0}\left(E_{N}\right)}{d E_{N}} .
$$

Then, assuming that $f_{\xi-s}\left(E_{N}\right)=$ const, the expected number of events (25) can be written as

$$
N_{s, 1 s h \exp }^{i}=N_{\exp }^{\mathrm{MB}}\left(\frac{V^{i}}{V^{\mathrm{MB}}}\right)\left(\frac{E_{N}^{\mathrm{MB}}}{E_{N}^{i}}\right)\left(\frac{f_{\xi-s}^{i}}{f_{1 s h}^{\mathrm{MB}}}\right)\left(\frac{\epsilon_{\xi-s}^{i}}{\epsilon_{1 s h}^{\mathrm{MB}}}\right)\left(\frac{\phi_{N}^{i}}{\phi_{N}^{\mathrm{MB}}}\right),
$$

where $V^{i}=A^{i} d^{i}$ is the volume of detector $i$ and $\phi_{N}^{i} \propto \phi_{\nu}^{i}$ is the integral flux of $N$ at a detector.

\section{Upscattering-decay, $U_{N} D_{\xi}$ scenario}

In this scenario (schematically shown in Fig. 2), $N$ is produced by the $\nu_{\mu}$ upscattering on material along a baseline and then it decays as $N \rightarrow \nu+\xi$. The $N$ decays inside a detector give an observable signal, while $N$ itself can be produced both in the detector and in the surrounding material. If $\lambda_{N} \gg d$, a large part of the $N$ flux can be formed outside a detector. The initial flux is the $\nu_{\mu}$ flux at the exit from the decay pipe, $d \phi_{\nu}^{0}\left(E_{\nu}\right) / d E_{\nu}$.

Let us first consider both production and sequential decay of $N$ inside a detector. Following the general formulas in Sec. III A, we obtain the number of $s$ events:

$$
N_{\xi-s}^{\mathrm{in}}=\epsilon V_{d} n_{d} \int d E_{N} f_{\xi-s}\left(E_{N}\right) \frac{d \phi_{N}^{\sigma}\left(E_{\nu}\right)}{d E_{N}} P_{\mathrm{dec}}^{\mathrm{in}}
$$

where $V_{d} \equiv A d$ and

$$
\frac{d \phi_{N}^{\sigma}\left(E_{N}\right)}{d E_{N}} \equiv \int d E_{\nu} \frac{d \phi_{\nu}^{0}\left(E_{\nu}\right)}{d E_{\nu}} \frac{d \sigma\left(E_{\nu}, E_{N}\right)}{d E_{N}}
$$

Notice that $n d \phi_{N}^{\sigma}\left(E_{N}\right) / d E_{N}$ is the density of $N$ flux produced in the detector. In the prefactor of Eq. (37), the product $A d n=V_{d} n=M_{d}$ gives the mass of a detector.

According to Fig. 2, the decay factor equals

$$
P_{\mathrm{dec}}^{\mathrm{in}}=\int_{l}^{l+d} \frac{d y}{d} \int_{y}^{l+d} \frac{d z}{\lambda_{N}} S_{N}(z-y),
$$

which gives explicitly

$$
P_{\mathrm{dec}}^{\mathrm{in}}=1-\frac{\lambda_{N}}{d}\left(1-e^{-d / \lambda_{N}}\right) .
$$

In the asymptotics, $\lambda_{N} \gg d$, this factor converges to

$$
P_{\mathrm{dec}}^{\mathrm{in}} \approx \frac{d}{2 \lambda_{N}},
$$

and, in the opposite case, $\lambda_{N} \ll d$, we have $P_{\text {dec }} \rightarrow 1$.

Let us find the contribution to the number of events in a detector from $N$ produced in surrounding material (dirt). We denote by $\Delta$ the distance between a detector and dirt (usually the air in a detector pit). For simplicity, we consider uniform surrounding medium with density $n_{b}$ and length $b$. Similarly to Eq. (37), the number of observable events equals

$$
N_{\xi-s}^{\text {out }}=\epsilon N_{b} \int d E_{N} \frac{d \phi_{N}^{\sigma}\left(E_{N}\right)}{d E_{N}} f_{\xi-s} P_{\mathrm{dec}}^{\text {out }}\left(E_{N}\right),
$$

where $N_{b}=n_{b} A b$ is the number of scatterers in medium. The decay factor $P_{\mathrm{dec}}^{\text {out }}$ differs from $P_{\mathrm{dec}}^{\text {in }}$ by limits of integration:

$$
P_{\mathrm{dec}}^{\mathrm{out}}=\int_{l_{p}}^{l_{p}+b} \frac{d y}{b} \int_{l_{p}+b+\Delta}^{l_{p}+b+\Delta+d} \frac{d z}{\lambda_{N}} S_{N}(z-y)
$$

which gives

$$
P_{\operatorname{dec}}^{\text {out }}\left(E_{N}\right)=\frac{\lambda_{N}}{b} e^{-\Delta / \lambda_{N}}\left(1-e^{-b / \lambda_{N}}\right)\left(1-e^{-d / \lambda_{N}}\right) .
$$

Here, $e^{-\Delta / \lambda_{N}}$ is the survival probability of $N$ between the end of dirt and the detector. If a detector and a pit have nonrectangular form, the parameters $\Delta$ and $d$ depend on the distance to the center (axis) of the setup $h$, and one needs to integrate over $h$.

In the limit $b \gg \lambda_{N}$, we obtain

$N_{\xi-s}^{\text {out }}=A n_{b} \epsilon \int d E_{N} \lambda_{N} \frac{d \phi_{N}^{\sigma}\left(E_{N}\right)}{d E_{N}} f_{\xi-s} e^{-\Delta / \lambda_{N}}\left(1-e^{-d / \lambda_{N}}\right)$. 
In this limit, the $N$ flux is collected along the distance of the order $\lambda_{N}$ in front of a detector.

The total number of events due to $N$ produced in a detector and surrounding materials can be written as

$$
N_{\xi-s}^{\mathrm{tot}}=N_{\xi-s}^{\mathrm{in}}+N_{\xi-s}^{\mathrm{out}}=A d n_{d} \epsilon \int d E_{N} \frac{d \phi_{N}^{\sigma}\left(E_{N}\right)}{d E_{N}} f_{\xi-s}\left(P_{\mathrm{dec}}^{\text {in }}+\frac{b n_{b}}{d n_{d}} P_{\mathrm{dec}}^{\text {out }}\right)
$$

or, explicitly,

$$
N_{\xi-s}^{\mathrm{tot}}=A d n_{d} \epsilon \int d E_{N} \frac{d \phi_{N}^{\sigma}\left(E_{N}\right)}{d E_{N}} f_{\xi-s}\left\{1+\frac{\lambda_{N}}{d}\left(1-e^{-d / \lambda_{N}}\right)\left[\frac{n_{b}}{n_{d}} e^{-\Delta / \lambda_{N}}\left(1-e^{-b / \lambda_{N}}\right)-1\right]\right\} .
$$

In the limit $b \gg \lambda_{N}$, the number of events equals

$$
N_{\xi-s}^{\mathrm{tot}}=A d n_{d} \epsilon \int d E_{N} \frac{d \phi_{N}^{\sigma}\left(E_{N}\right)}{d E_{N}} f_{\xi-s}\left[1+\frac{\lambda_{N}}{d}\left(1-e^{-d / \lambda_{N}}\right)\left(e^{-\Delta / \lambda_{N}} \frac{n_{b}}{n_{d}}-1\right)\right] .
$$

For $\lambda_{N}>d$ and $\Delta<\lambda_{N}$, the contribution from dirt can be several times larger than the one from a detector.

Let us consider the dependence of numbers of events (48) on $c \tau^{0}$. It is largely determined by the ratios of decay factors for the detector $i$ and MiniBooNE taken at certain effective energies $E_{N}^{\mathrm{MB}}$ and $E_{N}^{i}$. For the contribution due to $N$ production inside a detector $i$, the dependence of the number of events on $c \tau^{0}$ is determined by the ratio of decay factors $P_{\mathrm{dec}}^{\mathrm{in}}(40)$, which can be written as

$$
r_{\mathrm{dec}}=\frac{1-\frac{c \tau^{0}}{D^{i}}\left(1-e^{-D^{i} / c \tau^{0}}\right)}{1-\frac{c \tau^{0}}{D^{\mathrm{MB}}}\left(1-e^{-D^{\mathrm{MB}} / c \tau^{0}}\right)}
$$

where

$$
D^{i} \equiv d^{i} \frac{m_{N}}{E^{i}}
$$

are the "reduced" sizes of detectors $\left(d / \lambda=D / c \tau^{0}\right)$. Among the experiments we consider, MiniBooNE has the largest reduced size, $D^{\mathrm{MB}}>D^{i}$. Numerically, for MiniBooNE $\left(d_{\mathrm{MB}}=8 \mathrm{~m}\right.$ and $\left.E_{N}^{\mathrm{MB}}=0.8 \mathrm{GeV}\right)$, we obtain

$$
D_{\mathrm{MB}}=1.5 \mathrm{~m}\left(\frac{m_{N}}{0.15 \mathrm{GeV}}\right) \text {. }
$$

Taking this into account, we find the following from Eq. (49).

(i) For $c \tau^{0}<D^{i}$, both decay probabilities (for MiniBooNE and $i$ detector) are close to 1 , so that $r_{\mathrm{dec}} \approx 1$. Consequently, the ratio of number of events does not depend on $c \tau^{0}$ as well as on $m_{N}$. The dependence of the expected number of events on $m_{N}$ follows from fluxes and cross sections.

(ii) In the interval $D^{i}<c \tau^{0}<D^{\mathrm{MB}}, N$ still has space to decay in MiniBooNE and $P_{\mathrm{dec}}^{\mathrm{MB}} \sim 1$, while the $N$ decay length becomes larger than the $i$ detector length and, therefore, $P^{i}$ decreases. As a result, the number of $i$ detector events should decrease.

(iii) For $c \tau^{0}>D_{\mathrm{MB}}$, the particles $N$ decay only partially in both detectors, and the ratio of decay factors converges to

$$
r_{\mathrm{dec}}^{\infty}=\frac{P_{\mathrm{dec}}^{i}}{P_{\mathrm{dec}}^{M B}}=\frac{D^{i}}{D^{\mathrm{MB}}}=\frac{d^{i} E_{N}^{\mathrm{MB}}}{d^{\mathrm{MB}} E_{N}^{i}}
$$

Again, dependences of $r_{\mathrm{dec}}$ and prediction of the number of events on $c \tau^{0}$ as well as on $m_{N}$ disappear.

In the limit $c \tau^{0} \rightarrow 0$, the decay factors $P_{\mathrm{dec}} \approx 1$ and the number of events can be estimated as

$N_{\xi-s}^{\mathrm{ND}}=N_{1 s h, \exp }^{\mathrm{MB}}\left(\frac{M^{i}}{M^{\mathrm{MB}}}\right)\left(\frac{f_{\xi-s}^{\mathrm{ND}}}{f_{1 e}^{\mathrm{MB}}}\right)\left(\frac{\epsilon_{\xi-s}^{i}}{\epsilon_{1 s h}^{\mathrm{MB}}}\right)\left(\frac{\sigma^{i}}{\sigma^{\mathrm{MB}}}\right)\left(\frac{\phi_{\nu}^{i}}{\phi_{\nu}^{\mathrm{MB}}}\right)$,

as $\phi_{\nu}^{i} \propto(\mathrm{POT})^{i}[23]$.

For $N$ production in the dirt and then decay in a detector, we have

$$
r_{\mathrm{dec}}=\frac{\frac{\lambda_{N}^{i}}{d^{i}}\left(1-e^{-d^{i} / \lambda_{N}^{i}}\right) \frac{n_{b}^{i}}{n_{d}^{i}} e^{-\Delta^{i} / \lambda_{N}^{i}}\left(1-e^{-b^{i} / \lambda_{N}^{i}}\right)}{1+\frac{\lambda_{N}^{\mathrm{MB}}}{d^{\mathrm{MB}}}\left(1-e^{-d^{\mathrm{MB}} / \lambda_{N}^{\mathrm{MB}}}\right)\left[\frac{n_{b}^{\mathrm{MB}}}{n_{d}^{\mathrm{MB}}} e^{-\Delta^{\mathrm{MB}} / \lambda_{N}^{\mathrm{MB}}}\left(1-e^{-b^{\mathrm{MB}} / \lambda_{N}^{\mathrm{MB}}}\right)-1\right]}
$$

Now, the decay factor (44) is proportional to $\lambda_{N}$, and in the limit $c \tau^{0} \rightarrow 0$ the ratio (54) equals 
Upscattering-double-decay scenario $\mathrm{U}_{\mathrm{N}} \mathrm{D}_{\mathrm{B}} \mathrm{D}_{\xi}$

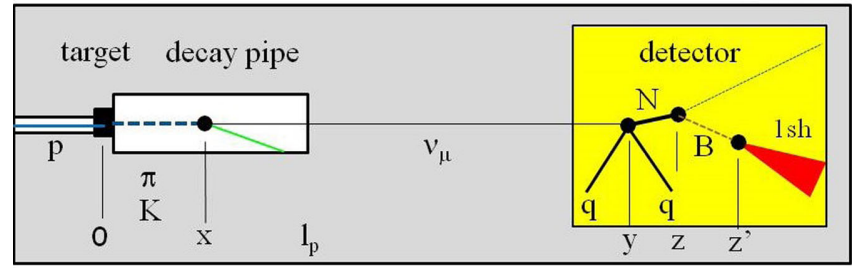

FIG. 3. Upscattering-double-decay scenario. Black blobs show the interaction points, the red triangle denotes the EM shower, and $l_{p}$ is the length of the decay pipe.

$$
r_{\mathrm{dec}}^{0}=\frac{\lambda_{N}^{i}}{d^{i}} \frac{n_{b}^{i}}{n_{d}^{i}}
$$

so that the contribution from dirt vanishes. In the opposite limit, $c \tau^{0} \rightarrow \infty$, we have

$$
r_{\mathrm{dec}}^{\infty}=\left(\frac{n_{d}^{\mathrm{MB}}}{n_{d}^{i}}\right)\left(\frac{n_{b}^{i}}{n_{b}^{\mathrm{MB}}}\right)\left(\frac{b^{i}}{b^{\mathrm{MB}}}\right)\left(\frac{\lambda_{N}^{\mathrm{MB}}}{\lambda_{N}^{i}}\right)
$$

That is, the dirt contribution converges to a constant.

\section{Upscattering-double-decay, $U_{N} D_{B} D_{\xi}$ scenario}

This scenario (schematically shown in Fig. 3) has three vertices with one $\nu_{\mu}$ upscattering and two sequential decays. The initial state and initial part are the same as in the previous scenario. When $B$ decays promptly, this scenario is similar to the $U_{N} D_{\xi}$ described in Sec. III C. In this case, the only but rather relevant difference is that the invariant mass of $\xi$ is fixed by the mass of a boson $B$ : $W_{\xi}=m_{B}$. The latter can be substantially smaller than the mass of $N$ which affects the signature factor. In Sec. V, we will show results for a short $B$ lifetime.

In what follows, we will consider the new contribution from $N$ production outside a detector. The number of expected events can be written as

$$
\begin{aligned}
N_{\xi-s}^{\text {out }}= & \epsilon N_{b} \int d E_{B} f_{\xi-s}\left(E_{B}\right) \\
& \times \int d E_{N} \frac{d \phi_{N}^{\sigma}\left(E_{N}\right)}{d E_{N}} \frac{d \Gamma_{N}\left(E_{N}, E_{B}\right)}{\Gamma_{N}^{\text {tot }} d E_{B}} P_{\operatorname{dec}}^{\text {out }}\left(E_{N}, E_{B}\right),
\end{aligned}
$$

where $d \phi_{N}^{\sigma} / d E_{N}$ was defined in Eq. (38) and additional integration was introduced over $d E_{B}$. The distribution $d \Gamma_{N}\left(E_{B}, E_{\xi}\right) / \Gamma_{N}^{\text {tot }} d E_{\xi}$ is included in $f_{\xi-s}\left(E_{B}\right)$. The decay factor is given by

$$
\begin{aligned}
P_{\mathrm{dec}}^{\mathrm{out}}\left(E_{N}, E_{B}\right)= & \int_{l_{p}}^{l_{p}+b} \frac{d y}{b} \int_{l}^{l+d} \frac{d z^{\prime}}{\lambda_{B}} \\
& \times \int_{y}^{z^{\prime}} \frac{d z}{\lambda_{N}} S_{N}(z-y) S_{B}\left(z^{\prime}-z\right) .
\end{aligned}
$$

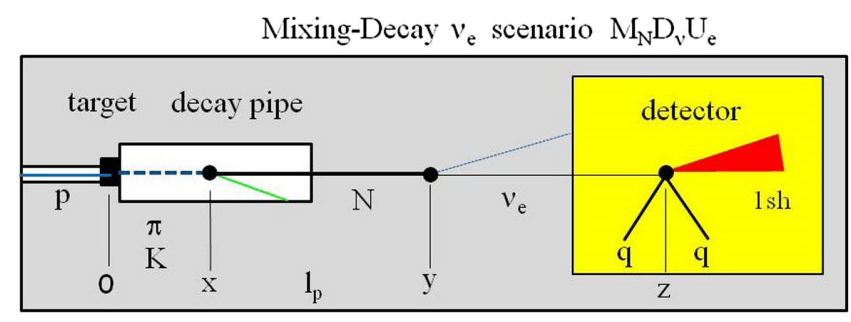

FIG. 4. The same as in Fig. 1 but for the mixing-decay into $\nu_{e}$ scenario.

Here, $S_{N}=e^{-(z-y) / \lambda_{N}}, S_{N}=e^{-\left(z^{\prime}-z\right) / \lambda_{B}}$, and the limits of integrations can be immediately read off from Fig. 3, but with the $\nu_{\mu}$ upscattering in the dirt. Explicit integration gives

$$
\begin{aligned}
P_{\mathrm{dec}}= & \frac{\lambda_{N}^{2}}{\left(\lambda_{N}-\lambda_{B}\right) b}\left[1-e^{\left.-b / \lambda_{N}\right)}\right]\left(1-e^{-d / \lambda_{N}}\right) \\
& +\frac{\lambda_{B}^{2}}{\left(\lambda_{B}-\lambda_{N}\right) b}\left[1-e^{\left.-b / \lambda_{B}\right)}\right]\left(1-e^{-d / \lambda_{B}}\right) .
\end{aligned}
$$

The decay factor is symmetric with respect to interchange $\lambda_{N} \leftrightarrow \lambda_{B}$. In the limit $\lambda_{B} \rightarrow 0$ (fast $B$ decay), it coincides

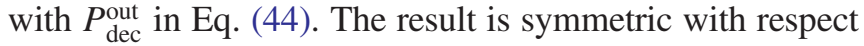
to $N$ and $B$.

If $\lambda_{B}=\lambda_{N}=\lambda$, we obtain

$$
P_{\mathrm{dec}}=\frac{2 \lambda}{b}\left(1-e^{-b / \lambda)}\right)\left(1-e^{-d / \lambda}\right)
$$

\section{E. Mixing-decay into $\nu_{e}, M_{N} D_{\nu} U_{e}$ scenario}

This scenario (schematically shown in Fig. 4) essentially provides an additional source of $\nu_{e}$ at low energies. Therefore, there is no restriction from angular dependence of the observed MiniBooNE events, but $N$ should be light enough to satisfy the timing bound. Therefore, it is dominantly produced in the $\pi$ decay.

Relatively light $N$ produced via mixing with $\nu_{\mu}$ decays into $\nu_{e}$ and a new light scalar or vector boson along the beam line: $N \rightarrow \nu_{e}+B$. In turn, the bosons $B$ may decay into a $\nu_{e} \bar{\nu}_{e}$ pair, thus enhancing the $\nu_{e}$ flux at low energies. Here, there are more interaction points in comparison to previous scenarios (although in one point the interactions are standard).

Since $N$ can decay already in the decay tunnel, the consideration should start from $\pi$ decay as in the $M_{N} D_{\xi}$ scenario in Sec. III B. In contrast to $M_{N} D_{\xi}$, decay of $N$ in a pipe does contribute to the observable signal, since $\nu_{e}$ are stable and can travel to a detector. This requires different consideration from $M_{N} D_{\xi}$.

The initial flux is the pion flux produced in a proton target $\phi_{\pi}^{0}$. Then using general formulas of Sec. III A we can write the expression for the number of expected events: 


$$
\begin{aligned}
N_{e-1 s h}= & \epsilon A d n_{d} \int d E_{\nu} \sigma^{C C}\left(E_{\nu}\right) f_{e-1 s h}\left(E_{\nu}\right) \\
& \times \int d E_{N} \frac{d \Gamma_{N}\left(E_{N}, E_{\nu}\right)}{\Gamma_{N}^{\text {tot }} d E_{\nu}} \\
& \times \int d E_{\pi} \frac{d \phi_{\pi}^{0}\left(E_{\pi}\right)}{d E_{\pi}} \frac{d \Gamma_{\pi N}\left(E_{\pi}, E_{N}\right)}{\Gamma_{\pi}^{\text {tot }} d E_{N}} P_{\operatorname{dec}}\left(\lambda_{\pi}, \lambda_{N}\right) .
\end{aligned}
$$

The decay factor equals

$$
P_{\mathrm{dec}}=\int_{l}^{l+d} \frac{d z}{d} \int_{0}^{l_{p}} \frac{d x}{\lambda_{\pi}} \int_{x}^{z} \frac{d y}{\lambda_{N}} e^{-x / \lambda_{\pi}} e^{-(y-x) / \lambda_{N}},
$$

and, explicitly,

$$
P_{\mathrm{dec}}=\left[1-e^{-l_{p} / \lambda_{\pi}}-g\left(\lambda_{\pi}, \lambda_{N}\right) \frac{\lambda_{N}}{d} e^{-b / \lambda_{N}}\left(1-e^{-d / \lambda_{N}}\right)\right],
$$

where

$$
g\left(\lambda_{\pi}, \lambda_{N}\right)=\left(1-\frac{\lambda_{\pi}}{\lambda_{N}}\right)^{-1}\left[e^{-l_{p} / \lambda_{N}}-e^{-l_{p} / \lambda_{\pi}}\right] .
$$

If $d \ll \lambda_{N}$, Eq. (61) reduces to

$$
P_{\mathrm{dec}} \approx\left(1-e^{-l_{p} / \lambda_{\pi}}-g\left(\lambda_{\pi}, \lambda_{N}\right) e^{-b / \lambda_{N}}\right) .
$$

Let us consider two limits of this result.

(i) $\lambda_{N} \rightarrow 0$ (very fast $N$ decay).-We have from Eq. (63)

$$
P_{\mathrm{dec}} \approx\left(1-e^{-l_{p} / \lambda_{\pi}}\right),
$$

which is nothing but the decay probability of pions in a pipe. It gives the $\nu_{\mu}$ flux at a detector.

(ii) $\lambda_{N} \rightarrow \infty$ (very slow $N$ decay). - In the lowest order in $l / \lambda_{N}$, we find from Eq. (63)

$$
P_{\mathrm{dec}} \approx\left(1-e^{-l_{p} / \lambda_{\pi}}\right) \frac{l^{\mathrm{eff}}}{\lambda_{N}},
$$

where $l^{\text {eff }}$ is the effective baseline:

$$
l^{\mathrm{eff}} \equiv b+l_{p}\left(1-e^{-l_{p} / \lambda_{\pi}}\right)^{-1}-\lambda_{\pi} .
$$

In the limits $\lambda_{\pi} \rightarrow 0$ and $\lambda_{\pi} \rightarrow \infty$, this equation gives $l^{\text {eff }}=$ $b+l_{p}$ and $l^{\text {eff }}=b$ correspondingly. For a typical situation with $\lambda_{\pi}=l_{p}$, we find from Eq. (64)

$$
l^{\text {eff }}=b+l_{p}(e-1)^{-1} \approx b+0.58 l_{p} .
$$

If $c \tau^{0} \rightarrow 0$, the ratio of decay factors converges to $r_{\mathrm{dec}}^{0}=1$, while for $c \tau^{0} \rightarrow \infty$

$$
r_{\mathrm{dec}}^{\infty}=\frac{E^{i}}{E^{\mathrm{MB}}} \frac{z^{i}\left(b^{i}+\lambda_{\pi}^{i}\right)+l_{p}^{i}}{z^{\mathrm{MB}}\left(b^{\mathrm{MB}}+\lambda_{\pi}^{\mathrm{MB}}\right)+l_{p}^{\mathrm{MB}}},
$$

where $z^{i} \equiv\left(1-e^{l_{p}^{i} / \lambda_{\pi}^{i}}\right)$. Consequently, in both limits the number of events does not depend on $c \tau^{0}$.

\section{F. Mixing-double-decay scenario, $M_{N} D_{B} D_{\xi}$}

According to this scenario, $N$ is produced in the $\pi$ and $K$ decays via mixing in $\nu_{\mu}$ within a decay pipe. Then $N$ decays along the baseline with emission of boson $B, N \rightarrow \nu+B$, and the latter decays $B \rightarrow \xi$ or $B \rightarrow \xi+B^{\prime}$. The $B$ decay should occur in a detector (see Fig. 5). This scenario reproduces various features of the previously described scenarios: In particular, for fast decaying $B, \lambda_{B} \ll d$, it is reduced to the $M_{N} D_{\xi}$ scenario.

The initial flux is the flux of pions (also $\mathrm{K}$ mesons) produced in the target. All three processes involved are decays. According to Fig. 5, the limits of integrations are the following: The coordinate of $\pi(K)$ decay is in the interval $x=\left[0-l_{p}\right]$; the coordinate of $N$ decay (and production of $B$ ) $y=[x-z]$; and the point of $B$ decay should be within the detector: $z=[l-(l+d)]$. With this, according to the general formulas in Sec. III A, the expression for the number of events can be written as

$$
\begin{aligned}
N_{\xi-s}= & \epsilon_{s}^{i} A \int d E_{\pi} \frac{\phi_{\pi}^{0}\left(E_{\pi}\right)}{d E_{\pi}} \int d E_{N} \frac{d \Gamma_{\pi N}\left(E_{\pi}, E_{N}\right)}{\Gamma_{\pi}^{\mathrm{tot}} d E_{N}} \\
& \times \int d E_{B} \frac{d \Gamma_{N}\left(E_{N}, E_{B}\right)}{\Gamma_{N}^{\mathrm{tot}} d E_{B}} f_{\xi-s}\left(E_{B}\right) P_{\mathrm{dec}}\left(E_{\pi}, E_{N}, E_{B}\right) .
\end{aligned}
$$

Here, the mixing parameter $\left|U_{\mu 4}\right|^{2}$ is included in $d \Gamma_{\pi} / d E_{N}$. The decay factor (24) equals

$$
\begin{aligned}
P_{\mathrm{dec}}\left(E_{\pi}, E_{N}, E_{B}\right)= & \int_{0}^{l_{p}} \frac{d x}{\lambda_{\pi}} \int_{l}^{l+d} \frac{d z}{\lambda_{B}} \\
& \times \int_{x}^{z} \frac{d y}{\lambda_{N}} e^{-x / \lambda_{\pi}} e^{-(y-x) / \lambda_{N}} e^{-(z-y) / \lambda_{B}} .
\end{aligned}
$$

Explicit integration over coordinates gives

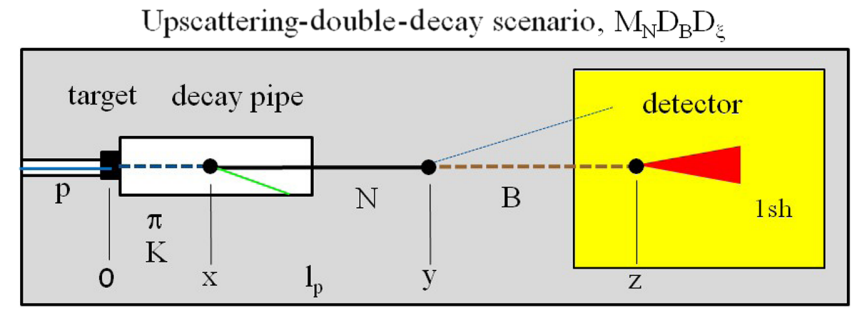

FIG. 5. The same as in Fig. 1 but for the mixing-double-decay scenario. 


$$
P_{\mathrm{dec}}=\frac{\lambda_{N}}{\lambda_{N}-\lambda_{B}} P_{M D}\left(\lambda_{N}\right)+\frac{\lambda_{B}}{\lambda_{B}-\lambda_{N}} P_{M D}\left(\lambda_{B}\right),
$$

where $\lambda_{B}=\left(E_{B} / m_{B}\right) c \tau_{B}^{0}$ and

$P_{M D}(\lambda)=\frac{1}{\left(1-\lambda_{\pi} / \lambda\right)} e^{-l / \lambda}\left[1-e^{-l_{p}\left(1 / \lambda_{\pi}-1 / \lambda\right)}\right]\left(1-e^{-d / \lambda}\right)$,

which coincides with the decay factor in the $M_{N} D_{\xi}$ scenario in Eq. (27). Notice that the expression in Eq. (69) is symmetric with respect to $\lambda_{N} \leftrightarrow \lambda_{B}$.

The scenario is determined by four parameters: $c \tau_{N}^{0}, m_{N}$, $c \tau_{B}^{0}$, and $m_{B}$. In the limit $\lambda_{B} \rightarrow 0$ (very fast $B$ decay), $P_{\mathrm{dec}} \rightarrow P_{\mathrm{dec}}^{N}\left(\lambda_{B}=0\right)$ and the latter coincides with expression (27) for the $M_{N} D_{\xi}$ scenario. In the limit $\lambda_{N} \rightarrow 0$ (very fast $N$ decay), $P_{\mathrm{dec}} \rightarrow P_{\mathrm{dec}}^{B}\left(\lambda_{N}=0\right)$. That is, we obtain the same expression (27) with just the substitution $\lambda_{N} \rightarrow \lambda_{B}$.

Let us consider the case $\lambda_{N}=\lambda_{B}$ which is reduced to two parameters and one expects the largest deviation from the result of the $M_{N} D_{\xi}$ scenario. In the limit $\lambda_{B} \rightarrow \lambda_{N}$, we can expand

$$
P_{M D}\left(\lambda_{B}\right)=P_{M D}\left(\lambda_{N}\right)+\left.\frac{d P_{M D}}{d \lambda_{B}}\right|_{\lambda_{B}=\lambda_{N}}\left(\lambda_{B}-\lambda_{N}\right) .
$$

Inserting this expression into Eq. (69) we find

$$
P_{\mathrm{dec}}(\lambda)=P_{M D}\left(\lambda_{N}\right)+\lambda \frac{d P_{M D}}{d \lambda}
$$

which gives

$$
\begin{aligned}
P_{\mathrm{dec}}= & P_{M D}(\lambda)\left[1-\frac{\lambda_{\pi}}{\lambda-\lambda_{\pi}}+\frac{l}{\lambda}+\frac{l_{p}}{\lambda} \frac{1}{e^{l_{p}\left(1 / \lambda_{\pi}-1 / \lambda\right)}-1}\right. \\
& \left.-\frac{d}{\lambda} \frac{1}{e^{d / \lambda}-1}\right] .
\end{aligned}
$$

For small size detector $d \ll \lambda$, we find

$$
P_{\mathrm{dec}} \approx P_{M D}(\lambda)\left[-\frac{\lambda_{\pi}}{\lambda-\lambda_{\pi}}+\frac{l}{\lambda}+\frac{l_{p}}{\lambda} \frac{1}{e^{l_{p}\left(1 / \lambda_{\pi}-1 / \lambda\right)}-1}\right] .
$$

If $\lambda_{\pi} \ll \lambda$, it can be rewritten as

$$
P_{\mathrm{dec}} \approx P_{M D}(\lambda) \frac{L\left(\lambda, l, l_{p}\right)}{\lambda},
$$

where

$$
L\left(l, l_{p}\right)=\left(l+\frac{l_{p}}{e^{l_{p} / \lambda_{\pi}}-1}-\lambda_{\pi}\right)
$$

The more precise expression weakly depends on $\lambda$, and in the first approximation $L=l$.

Using similar approximations in $P_{M D}(\lambda)$, we obtain explicitly

$$
P_{\mathrm{dec}} \approx h \frac{L}{\lambda} \frac{d}{\lambda} e^{-l / \lambda}
$$

and $h \approx 1-e^{l_{p} / \lambda_{\pi}} \approx 1$. The ratio of the decay factors (76) for a given detector $i$ and MiniBooNE can be written as

$$
\frac{P_{\mathrm{dec}}^{i}}{P_{\mathrm{dec}}^{\mathrm{MB}}}=\left(\frac{d^{i}}{d^{\mathrm{MB}}}\right)\left(\frac{L^{i}}{L^{\mathrm{MB}}}\right)\left(\frac{E^{\mathrm{MB}}}{E^{i}}\right)^{2} \exp \left(l^{\mathrm{MB}} / \lambda^{\mathrm{MB}}-l^{i} / \lambda^{i}\right) .
$$

As in the $M_{N} D_{\xi}$ scenario, the dependence of number of events on $c \tau^{0}$ shows up via the exponential upturn determined by the MiniBooNE parameters $l^{\mathrm{MB}}$ and $\lambda^{\mathrm{MB}}$ and constant asymptotics for large $c \tau^{0}$. The difference in comparison to the $M_{N} D_{\xi}$ scenario is the appearance of the additional factor

$$
\frac{L^{i}}{L^{\mathrm{MB}}} \frac{E^{\mathrm{MB}}}{E^{i}}
$$

For ND280 this factor equals 0.4 .

So, in all these special cases $P_{\text {dec }}$ are reduced to the twoparameter expression (27).

\section{G. Upscattering-decay into $\nu_{e}$ scenario, $U_{N} D_{\nu} U_{e}$}

Here, $N$ is produced via the $\nu_{\mu}$ upscattering (point $x$ ) outside the decay pipe (see Fig. 6). It decays into $\nu_{e}$ (the point $y$ ) and new light scalar or vector boson $N \rightarrow \nu_{e}+B$. Then $\nu_{e}$ via the CC interactions produces an electron in a detector (point $z$ ).

It is similar to the $M_{N} D_{\nu} U_{e}$ scenario, where the $N$ production via mixing is substituted by $\nu_{\mu}$ upscattering. That can bring a smallness as we discussed in Sec. II. In contrast to the $M_{N} D_{\nu} U_{e}$ scenario, here there is no production of $N$ in a decay pipe. There are two standard model (SM) vertices with production of $\nu_{\mu}$ and upscattering of $\nu_{e}$. The nonstandard interactions appear in production and decay of $N$.

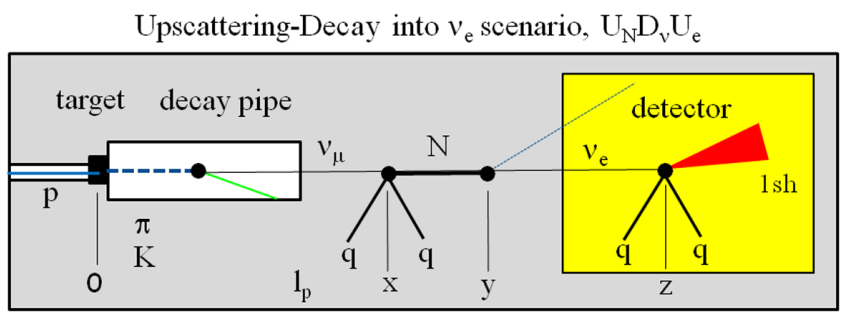

FIG. 6. The same as in Fig. 1 but for the upscattering-decay into $\nu_{e}$ scenario. 
Since $N$ production via the $\nu_{\mu}$ upscattering occurs outside a decay pipe, we can use the $\nu_{\mu}$ flux at the exit from the pipe $d \phi^{0}\left(E_{\nu_{\mu}}, l_{p}\right) / d E_{\nu_{\mu}}$ as the initial flux. Therefore, according to the general consideration in Sec. II A, the number of events can be written as

$$
\begin{aligned}
N_{e-s}= & \epsilon A \int d E_{\nu_{\mu}} \frac{d \phi_{\nu_{\mu}}^{0}\left(E_{\nu_{\mu}}\right)}{d E_{\nu_{\mu}}} \int d E_{N} \frac{d \sigma\left(E_{\nu_{\mu}}, E_{N}\right)}{d E_{N}} n_{N} l_{N} \\
& \times \int d E_{\nu_{e}} \frac{d \Gamma_{N}\left(E_{N}, E_{\nu_{e}}\right)}{\Gamma_{N}^{\text {tot }} d E_{\nu_{e}}} \sigma\left(E_{\nu_{e}}\right) n_{d} d f_{e-s}\left(E_{\nu_{e}}\right) P_{\mathrm{dec}}
\end{aligned}
$$

Here, $n_{N}$ and $l_{N}$ are the density and the length, respectively, of a layer in which $N$ production occurs. In Eq. (79), we used the integrated $\nu_{e}$ cross section and effective (integrated) signature factor, without introducing dependences on the electron energy. Since only one unstable particle $(N)$ is involved, the decay factor $P_{\mathrm{dec}}$ depends on a single survival probability $S_{N}(y-x)$.

There are two contributions to the total number of events related to the $N$ production in dirt (outside a detector) and in a detector. For simplicity, we consider dirt as a uniform medium with density $n_{b}$. For the first contribution, we use $l_{N}=b$ and $n_{N}=n_{b}$, and, consequently, the propagation factor equals

$$
P_{\mathrm{dec}}^{\mathrm{out}}=\int_{l_{p}}^{l} \frac{d x}{b} \int_{l}^{l+d} \frac{d z}{d} \int_{x}^{z} \frac{d y}{\lambda_{N}} e^{-(y-x) / \lambda_{N}}
$$

Explicit integration gives

$$
P_{\mathrm{dec}}^{\text {out }}=1-\frac{\lambda_{N}^{2}}{b d}\left(1-e^{-b / \lambda_{N}}\right)\left(1-e^{-d / \lambda_{N}}\right) .
$$

For the $N$ production in a detector, $l_{N}=d$ and $n_{N}=n_{d}$, and the limits of integration differ from those in Eq. (80):

$$
P_{\mathrm{dec}}^{\mathrm{in}}=\frac{1}{d^{2}} \int_{l}^{l+d} d x \int_{x}^{l+d} \frac{d y}{\lambda_{N}} \int_{y}^{l+d} d z e^{-(y-x) / \lambda_{N}}
$$

Integration gives

$$
P_{\mathrm{dec}}^{\mathrm{in}}=\frac{1}{2}-\frac{\lambda_{N}}{d}+\frac{\lambda_{N}^{2}}{d^{2}}\left(1-e^{-d / \lambda_{N}}\right) .
$$

In the limit of very fast $N$ decay, $\lambda_{N} \rightarrow 0$, the propagation factors converge to $P_{\mathrm{dec}}^{\text {out }} \rightarrow 1$ and $P_{\mathrm{dec}}^{\text {in }} \rightarrow 1 / 2$. In the opposite limit, $\lambda_{N} \rightarrow \infty$, both factors vanish: $P_{\mathrm{dec}}^{\text {out }}, P_{\mathrm{dec}}^{\text {in }} \rightarrow 0$.

The sum of two contributions (from "out" and "in" production) is proportional to

$$
n_{b} n_{d} b d\left(P_{\mathrm{dec}}^{\mathrm{out}}+\frac{n_{d}}{n_{b}} \frac{d}{b} P_{\mathrm{dec}}^{\mathrm{in}}\right)
$$

Decay factors similar to Eq. (81) appear in the $U_{N} D_{\xi}$ scenario [see Eqs. (49) and (52)]. The difference is that in the $U_{N} D_{\xi}$ scenario $N$ decays immediately into the observed particles $D_{\xi}$, and, therefore, the decay should occur in a detector. In the present scenario, $\xi=e$ is produced in two steps $D_{\nu} U_{e}$, and, therefore, $N$ can decay both in the detector and in the dirt.

Let us consider $c \tau_{0}$ dependence of the number of events. Since $n_{b} b \gg n_{d} d$, in the first approximation the $N$ production in a detector can be neglected. Then according to Eq. (81) there are two characteristic scales in the setup: $b$ and $d$, which correspond to two regions of the $\nu_{\mu}$ upscatterings followed by decays.

In the limit $\lambda_{N} \gg b, d$, Eq. (81) gives

$$
P_{\mathrm{dec}}^{\mathrm{out}} \approx \frac{b+d}{\lambda_{N}} \approx \frac{b}{\lambda_{N}} .
$$

In the intermediate range $d \ll \lambda_{N} \ll b$, we obtain $P_{\mathrm{dec}}^{\text {out }} \approx 1-\lambda_{N} / b$, and for very fast decay, $\lambda_{N} \ll b, d$, $P_{\mathrm{dec}}^{\text {out }} \approx 1-\lambda_{N}^{2} / b d$. The ratio of the decay factors for a given experiment and MiniBooNE has the following dependence on $c \tau_{0}$. In the asymptotics $c \tau_{0} \gg b^{\mathrm{MB}} m_{N} / E^{\mathrm{MB}}$, the ratio is constant:

$$
r^{i} \equiv \frac{P_{\mathrm{dec}}^{\mathrm{out}, i}}{P_{\mathrm{dec}}^{\mathrm{out}, \mathrm{MB}}} \approx \frac{b^{i}}{b^{\mathrm{MB}}} \frac{E^{\mathrm{MB}}}{E^{i}},
$$

and for experiments under consideration, $r^{i}<1$. With a decrease of $c \tau_{0}$, the ratio increases mainly in the intermediate region $d^{i} m_{N} / E^{i}<c \tau_{0}<b^{\mathrm{MB}} m_{N} / E^{\mathrm{MB}}$ and then converges to 1 at $c \tau_{0}<d^{i} m_{N} / E^{i}$. So, qualitatively, the dependence is similar to the dependence for other upscattering scenarios with, however, a longer transition region between the two asymptotics.

We described this scenario for completeness. It will be difficult (if possible) to construct a viable model that matches this scenario. Indeed, here there are two $\left(\nu_{\mu}\right.$ and $\left.\nu_{e}\right)$ upscattering vertices which bring smallness to the number of events. Furthermore, the transitions can be treated as flavor-violating nonstandard interactions (NSIs) that transform $\nu_{\mu}$ to $\nu_{e}$, and there are stringent bounds on this NSI. Therefore, in what follows, we will not present detailed phenomenological studies of this scenario.

\section{SIGNATURE FACTORS, CROSS SECTIONS, EXPERIMENTS, AND BOUNDS}

The key idea is that new physics scenarios that explain the MiniBooNE excess should produce visible numbers of events in the near detectors of various neutrino experiments. That will allow us to put bounds on the scenarios. 
Here, we describe the relevant features of different experiments as well as the theoretical and experimental results. We compute the upper limits on numbers of events due to new physics.

\section{A. Signal}

The observable signal is given by a deposit of electromagnetic energy from a final state $\xi$. Depending on the particle ID capabilities of a detector $i$, a given state $\xi$ can be (mis)identified with a number of other particle states. Associated with this identification are detector and analysis efficiencies. Below, we describe our approach for quantifying this. We also discuss the cross section input used for the upscattering scenarios.

\section{Efficiency}

The experiments $i$ quote signature efficiencies for the signatures $s^{i}$ which are, in general, a product of a detector efficiency $\epsilon_{\xi}^{i}$, a particle (mis)identification efficiency $f_{\xi-s^{i}}^{i}$, and signal selection efficiency $\epsilon_{s^{i}}^{i}$.

The detector efficiency $\epsilon_{\xi}^{i}$ quantifies the probability that a final state $\xi$ is registered in any way. In what follows, we assume that $\epsilon_{\xi}^{i}=1$. The misidentification efficiency or the signature factor $f_{\xi-s^{i}}^{i}$ is the fraction of cases when final state $\xi$ produces a signature $s^{i}$. The signal selection efficiency $\epsilon_{s^{i}}^{i}$ quantifies the so-called quality cuts (which include kinematic cuts) of the events that are needed to enhance the signal-over-background ratio. These efficiencies depend strongly on the considered signatures, and we take their values from experiments.

\section{Signature factor}

In general, the signature factor includes an integration over the phase space of kinematical variables and (mis) identification factors $I_{e}^{i}$, which depend on the type of detector.

Some detectors can distinguish events induced by a single photon, an $e^{+} e^{-}$pair, from those induced by a single electron. This is usually accomplished via measuring the energy loss $d E / d x$ over the whole trajectory or only in its initial part (like in MINER $\nu \mathrm{A}$ ). Detectors that have a magnetic field, like NOMAD or T2K ND280, also use the bending of tracks for particle ID.

We can introduce the signature factors in a different way considering final interactions (scattering or decay) which produce the state $\xi$. Then $f$ can be defined as the fraction of the final interactions in which the event $s^{i}$ appears. Formally, that implies summation over $\xi$.

Let us consider first scattering. For electrons that are produced by the CCQE $\nu_{e}$ scattering on nucleons $(\xi=e)$, we can write

$$
f_{e-s^{i}}\left(E_{\nu}\right)=\int_{E_{e}^{\mathrm{th}}} d E_{e} I_{s^{i}}\left(E_{e}\right) \frac{1}{\sigma^{\mathrm{tot}}} \frac{d \sigma\left(E_{\nu}, E_{e}\right)}{d E_{e}},
$$

where $I_{s^{i}}\left(E_{e}\right)$ is the probability that the electron with energy $E_{e}$ will show up as the $s^{i}$ event. In experiments capable to disentangle showers induced by $\gamma$ and $e$, the factor $I_{e-1 s h}\left(E_{e}\right) \sim 1$, which then leads to $f_{e-1 s h} \approx 1$.

Let us consider final states $\xi$ that originate from $N$ or $B$ decays. For $\xi=\gamma$,

$f_{\gamma-1 s h}^{i}\left(E_{N}\right)=\int d E_{\gamma} \frac{1}{\Gamma_{N}\left(E_{N}\right)} \frac{d \Gamma_{N}\left(E_{N}, E_{\gamma}\right)}{d E_{\gamma}} I_{\gamma-1 s h}\left(E_{\gamma}\right)$.

Again, if $I_{\gamma-1 s h}\left(E_{\gamma}\right) \approx 1$, the definition (84) gives $f_{\gamma-1 s h}^{i} \approx 1$.

In general, the signature factor for an $s^{i}$ event can be written as

$$
\begin{aligned}
f_{\xi-s^{i}}^{i}\left(E_{N}, m_{N}\right)= & \frac{1}{\Gamma_{N}\left(E_{N}, m_{N}\right)} \\
& \times \int^{\Pi_{s}^{i}} d \Pi_{\xi} \frac{d \Gamma_{N}\left(E_{N}, m_{N}, \Pi_{\xi}\right)}{d \Pi_{\xi}} I_{\xi-s^{i}}\left(\Pi_{\xi}\right),
\end{aligned}
$$

where $\Pi_{s^{i}}$ is the final state phase space in which the produced state $\xi$ shows up as an $s^{i}$ event in the experiment $i$.

For the final state being $\nu \gamma(\xi=\gamma)$, the relevant phase space is above the energy threshold, which is, for instance, $E_{\gamma}>100 \mathrm{MeV}$ in MiniBooNE (used to suppress cosmic ray backgrounds). In experiments without the $\gamma-e$ identification, and for high energies of $N, \Pi_{\gamma}^{i}$ is nearly the entire phase space. Thus, $f_{\gamma-1 s h}^{i}\left(E_{N}, m_{N}\right) \approx 1$.

The $e^{+} e^{-}$pair $\left(\xi=e^{+} e^{-}\right)$can produce two-shower (2e showers) events as well as single-shower events, if one of the components is missing or if two components are nearly collinear. For several detectors, the unique relevant criterion for differentiation between the single- and double-shower events is the invariant mass of the pair, $W_{e e}$. If $W_{e e}<W_{c}$, where $W_{c}$ is a certain critical value, the pair shows up as a single-shower event, while for $W_{e e}>W_{c}$ as the twoshower event. This means that $I_{e e-1 s h}\left(W_{e e}\right)=1$ when $W_{e e}<W_{c}$ and $I_{e e-1 s h}\left(W_{e e}\right)=0$ when $W_{e e}>W_{c}$.

When the $e^{+} e^{-}$pair is created from the three-body decay $N \rightarrow \nu e^{+} e^{-}, W_{e e}$ is not fixed and one needs to use the function $I_{e e-1 s h}\left(W_{e e}\right)$. The steplike $I_{e e-1 s h}\left(W_{e e}\right)$ determines the limits of integration. The fraction of decays with $W_{e e}<W_{c}$, which appear as a single-shower event, equals 


$$
f_{e e-1 s h}^{i}\left(x, m_{N}\right)=\frac{1}{\Gamma\left(N \rightarrow \nu e^{+} e^{-}\right)} \int_{0}^{W_{c}} d W_{e e} \frac{d \Gamma\left(N \rightarrow \nu e^{+} e^{-}\right)}{d W_{e e}}=\frac{W_{c}^{8}+2 W_{c}^{2} m_{N}^{6}-2 W_{c}^{6} m_{N}^{2}}{m_{N}^{8}} .
$$

We take $W_{c}=30 \mathrm{MeV}$ for MiniBooNE [24] and $W_{c}=$ $5 \mathrm{MeV}$ for the T2K near detector ND280 (cf. Ref. [25]), and we estimate $W_{c}=30 \mathrm{MeV}$ for PS191. For other detectors, we do not use an invariant mass threshold for our analysis; i.e., we assume that $e^{+} e^{-}$pairs and photons give the same signature. Notice that $f$ defined in this way does not depend on $E_{N}$, which simplifies computations.

If the $e^{+} e^{-}$pair appears from the two-body decay of a new boson, $B \rightarrow e^{+} e^{-}$, the invariant mass $W_{e e}$ is fixed: $W_{e e}=m_{B}$. Therefore, the signature factor is determined uniquely by the mass of $B$ : For $m_{B}<W_{c}$, we have $f_{e e-1 s h}^{i}=1$, while for $m_{B}>W_{c}, f_{e e-1 s h}^{i}=0$. This is realized, e.g., in scenarios with the decay chain $N \rightarrow \nu B, B \rightarrow e^{+} e^{-}$, where an on-shell dark photon $B$ is produced. For the two-shower signature, we have relation $f_{e e-2 s h}^{i}=1-f_{e e-1 s h}^{i}$.

\section{B. Cross sections and fluxes}

In the presence of new physics, the cross sections of heavy or light neutrino interactions depend on the specific model of interactions, i.e., on the mass of the mediator, Lorentz structure of coupling, etc. Since we compute the ratios of numbers of events, the model dependence of the cross sections mostly cancels.

However, there are still some uncertainties that depend on the nature of the new particle mediating upscattering. We find that for ND280 such uncertainty is at most at the level of $\sim 20 \%$. This value was obtained by comparing the predicted number of events for the vector and scalar mediators at $c \tau^{0} \rightarrow 0$. The reason for this small uncertainty at ND280 (as well as at PS191) is that the flux of neutrinos peaks at $\sim 1 \mathrm{GeV}$ as is the case for MiniBooNE. Therefore, the cross sections should be taken at similar energies, and the effect of change from vector to scalar mediator also cancels in the ratio Eq. (18).

The situation is different with MINER $\nu \mathrm{A}$, where the spin of the mediator matters. The typical energy of neutrino flux at MINER $\nu \mathrm{A}\left(E^{\mathrm{MV}}\right)$ is a few $\mathrm{GeV}$ higher than that at MiniBooNE $\left(E^{\mathrm{MB}}\right)$. Depending on whether the mediator is a new light vector or a scalar, the cross section grows or decreases in this energy range between $E^{\mathrm{MB}}$ and $E^{\mathrm{MV}}$. Namely, in the scalar mediator case of models $[11,12]$, the cross section decreases with energy substantially, so that $\mathrm{MINER} \nu \mathrm{A}$ is not able to probe such a scenario, contrary to the case of a vector mediator [26]. Keeping in mind this result for MINER $\nu$ A, we will mainly consider the vector mediator cases. Constraints from all experiments apart from $\operatorname{MINER} \nu \mathrm{A}$ are roughly independent of the nature of the mediator, as argued above. $\mathrm{NO} \nu \mathrm{A}$ also has the flux peaking at a larger energy than the MiniBooNE flux (given the same beam as for MINER $\nu \mathrm{A}$ ). Therefore, we use results from $\mathrm{NO} \nu \mathrm{A}$ to probe only a scenario where upscattering goes via SM charged current process $\left(M_{N} D_{\nu} U_{e}\right)$.

Furthermore, to cover all the possibilities, we consider both partially coherent and incoherent interactions. For the partially coherent case, we take the mass of mediator in the upscattering process to be $30 \mathrm{MeV}$ in accord with the benchmark point of Ref. [10]. For the incoherent case, we calculate the cross section for the mediator mass of $1.25 \mathrm{GeV}$ (using the cookbook presented in Ref. [27]), which corresponds to the benchmark point in Ref. [7]. For the quasielastic scattering of $\nu_{e}$, we use the $\nu_{\mu}$ upscattering cross section from Ref. [28] as a proxy. Differences of the cross sections due to difference of the electron and muon masses should be minor, because they are both small compared to the neutrino energies.

\section{Experiments and bounds}

\section{MiniBooNE}

Some information on MB has already been presented in Sec. II. The total number of muon neutrinos that passed through the MiniBooNE detector in positive (negative) horn polarity mode is $8.12 \times 10^{17}\left(3.1 \times 10^{17}\right)$ [29]. This corresponds to the muon neutrino flux per POT:

$$
\phi^{\mathrm{MB}}=5.19 \times 10^{-10} \mathrm{~cm}^{-2}(\mathrm{POT})^{-1} .
$$

The relevant parameters of the experimental setup are the decay pipe length $l_{p}^{\mathrm{MB}}=50 \mathrm{~m}$, baseline $l^{\mathrm{MB}}=540 \mathrm{~m}$, average detector length $d^{\mathrm{MB}}=8 \mathrm{~m}$, and the target mass $m^{\mathrm{MB}}=800 \mathrm{t}$. The average electron reconstruction and selection efficiency is $\epsilon_{1 s h}^{\mathrm{MB}} \simeq 10 \%$. Taking this average value of the efficiency instead of using the energy-dependent efficiencies [30] introduces a negligible $2 \%$ effect.

Apart from single-shower events, MiniBooNE observed also the two-shower events, and this can be a powerful probe of scenarios with $\xi=e e$ and $\xi=\gamma \gamma$. We have, however, estimated that this gives weaker bounds on the scenarios than the two-shower data from ND280.

\section{2. $T 2 K ~ N D 280$}

The T2K ND280 (ND280 for brevity) is sourced by $30 \mathrm{GeV}$ protons that interact with the graphite target [31]. The lengths involved are $l_{p} \simeq 100 \mathrm{~m}, b=230 \mathrm{~m}$ (dirt), and $l^{\mathrm{ND}}=280 \mathrm{~m}$ [32].

$\mathrm{ND} 280$, placed at $2.5^{\circ}$ off axis, is a multicomponent detector which consists of the following main subdetectors. 
(i) The $\pi^{0}$ detector POD.-The P0D filled with water has a target mass $m_{P 0 D}^{\mathrm{ND}}=15.8 \mathrm{t}$ and a length $d_{P 0 D}^{\mathrm{ND}}=2 \mathrm{~m}[33]$.

(ii) The tracking detector containing the three time projection chambers (TPCs) filled in by Ar gas.Each TPC module has a mass of $0.3 \mathrm{t}$ and a length of $0.9 \mathrm{~m}$.

(iii) Two fine-grained detectors (FGDs) filled in by scintillators.-The mass and the length of each FGD are $1.1 \mathrm{t}$ and $0.365 \mathrm{~m}$, correspondingly [34]. The detectors are magnetized with a field strength of $0.2 \mathrm{~T}$, which, together with energy loss tracking, allows for a very good particle identification capacity. The distance between the downstream edge of POD and the upstream edge of FGD1 equals $\Delta^{\mathrm{ND}}=1 \mathrm{~m}$.

Strictly, one has to consider interactions, decays, and detection in all these detectors separately. For simplicity, we will neglect most of the detector substructures. The neutrino flux is taken from Ref. [35]. We use two datasets from two independent studies: a search for heavy neutrinos [36] and an analysis of electron neutrino CCQE [23]. The latter gives bounds on numbers of $\gamma$ showers and $e$ showers.

(a) Resolved $e^{+} e^{-}$pairs: two showers.- $-\mathrm{T} 2 \mathrm{~K}$ searched the resolved $e^{+}$and $e^{-}$tracks (showers) from hypothetical heavy neutrino decays inside the TPC in Ref. [36]. In this study, $12.34 \times 10^{20}\left(6.29 \times 10^{20}\right)$ POT in neutrino (antineutrino) mode were used. The selected events consist of two tracks of opposite charge originating from a vertex in TPC, without other tracks being observed in the TPC itself or in the detector located directly upstream (including P0D). This gives an effective detector length of $2.7 \mathrm{~m}$. The invariant mass of the two-track system was restricted by $W_{e e}<700 \mathrm{MeV}$ and the angle between two tracks $<90^{\circ}$. The angle between the system of the tracks and the beam axis for events passing selection criteria should be $\cos \theta>0.99$. To implement this cut in computations of numbers of events, we performed our own Monte Carlo simulation of final state angular distributions.

For the indicated number of POTs, the number of observed $e^{+} e^{-}$shower events in neutrino mode, which satisfy the selection criteria, equals $N_{e e}^{\mathrm{ND}_{\nu}, \text { obs }}=62$. The expected number of events from the standard sources (various neutrino interactions) is $N_{e e}^{\mathrm{ND} \text {,th }}=58 \pm 2.8$. In the antineutrino mode, $N_{e e}^{\mathrm{ND}_{\bar{i}} \text {,obs }}=16$ events have been observed, while $N_{e e}^{\mathrm{ND} \text {,th }}=15.1 \pm 1.6$ are expected. We sum the events from both modes. We neglect the small error in the theory prediction (2.8) and combine the statistical uncertainty $\Delta N^{\text {stat }}=8.8$ with the systematic one in quadrature. For the latter, we take $15 \%$ relative uncertainty on the total number of observed events, which gives $\Delta N^{\text {syst }}=$ 11.7 (in what follows, for experiments where systematic uncertainty is not explicitly quoted, we assume the uncertainty of 15\%). With this, the following upper limits on a contribution from new physics are obtained:

$$
N_{2 s h}^{\mathrm{ND}}<20(1 \sigma), 34(2 \sigma), 49(2 \sigma) .
$$

Because of the particle ID capacity of ND280, the selected events can be produced by the $e^{+} e^{-}$pair only. We take the signature factor according to Eq. (86) for the three-body $N$ decay and $f_{e e-2 s h}=1$ for the two-body $B$ decay if $m_{B}>5 \mathrm{MeV}$.

(b) Unresolved (collinear) $e^{+} e^{-}$: one-shower events.The $\nu_{e}$ CCQE interactions were detected as isolated $e$ shower events [23]. The photon background is the most important for these events. In this connection, T2K studied single photons converted into $e^{+} e^{-}$pairs in the FGD1. The event selection criteria in the analysis include the following: Two tracks originate from the vertex in FGD1, and the energy losses in the tracks, $d E / d x$, are compatible with electrons. The tracks correspond to particles of opposite sign. The invariant mass is less than $W_{e e}<55 \mathrm{MeV}$ (the latter was imposed to ensure that $e^{+} e^{-}$originate from photon conversion). As signature efficiency, we adopt $\epsilon_{\gamma}^{\mathrm{ND}}=0.3$ from Ref. [23].

Total numbers of events of this type $N_{\gamma}^{\mathrm{ND}, \text { obs }}=647,182$, and 157 were found in the analysis of the forward horn current (FHC) data, the electron analysis of reverse horn current (RHC) data, and positron analysis of RHC data correspondingly. The simulated numbers of events that originate from SM processes (CCQE neutrino-nucleon scattering, resonant pion production, deep inelastic scattering, final state interactions of hadrons produced, etc.) turn out to be larger: $N_{\gamma}^{\mathrm{ND} \text {,th }}=700.97$ (FHC), 193.73 (electron RHC), and 169.31 (positron RHC).

We sum up the event numbers from FHC and the positron RHC data. ${ }^{5}$ The statistical error on the combined event numbers, $\Delta N^{\text {stat }}=28.1$, and the $15 \%$ systematic error, $\Delta N^{\text {syst }}=118.8$, are summed in quadrature. This gives the upper bounds on numbers of isolated $\gamma$ 's from new physics:

$$
N_{\gamma}^{\mathrm{ND}}<58(1 \sigma), 181(2 \sigma), 305(3 \sigma) .
$$

The deficit of observed signal events with respect to the prediction strengthen the bound. Here, signature factor $f_{\gamma-1 s h}^{\mathrm{ND}}=1$.

We will not use results of a dedicated search for the single-photon events at T2K ND280 in Ref. [25] due to low statistics.

(c) Single e shower. In the same ND280 study of the $\nu_{e^{-}}$ CCQE interactions in Ref. [23], the total numbers of 697, 176, and 95e-like events were found in the FHC, electron

\footnotetext{
${ }^{5}$ Including also the electron analysis would add information, but we have to take the correlation of the two analyses into account to which we have no access.
} 
RHC, and positron RHC analyses, respectively. These numbers are smaller than the expected numbers from various standard neutrino interactions: $797,175.92$, and 99.99. As before, we combine the event numbers from the FHC mode and the positron RHC mode. The statistical error $\Delta N^{\text {stat }}=28.3$ and the $15 \%$ relative systematic error $\Delta N^{\text {syst }}=120.6$ are added in quadrature. This leads to the upper bounds on numbers of $e$-like events from new physics:

$$
N_{e}^{\mathrm{ND}}<17(1 \sigma), 139(2 \sigma), 261(3 \sigma) .
$$

This analysis can be used to constrain scenarios with $\xi=e$. The reconstruction (and selection) efficiency for the $e$-like events equals $\epsilon_{e-s h}^{\mathrm{ND}}=0.3$ according to Ref. [23]. Notice that in future phases of experiment the T2K ND280 can substantially improve these bounds.

\section{MINER $\nu \mathrm{A}$}

The MINER $\nu \mathrm{A}$ experiment employs the mine injector beam line, where $120 \mathrm{GeV}$ protons hit a graphite target. The produced neutrino flux has variable energy in the range (2-20) GeV. We use two energy samples: ME (medium energy) with the peak at $E_{\nu}^{\mathrm{MV}}=6 \mathrm{GeV}$ and $\mathrm{LE}$ (low energy) with the peak at $E_{\nu}^{\mathrm{MV}}=4 \mathrm{GeV}$. The flux of usual neutrinos is substantially larger than the MB flux:

$$
\phi^{\mathrm{MV}, \mathrm{ME}}=3 \times 10^{-8} \mathrm{~cm}^{-2}(\mathrm{POT})^{-1} .
$$

The ratio of fluxes per POT $\phi_{\nu}^{\mathrm{MV}, \mathrm{ME}} / \phi_{\nu}^{\mathrm{MB}}=15$.

The experimental setup has the following sizes: $l_{p}^{\mathrm{MV}}=675 \mathrm{~m}, l^{\mathrm{MV}}=935 \mathrm{~m}$, and $d^{\mathrm{MV}}=3 \mathrm{~m}$; the target mass equals $m^{\mathrm{MV}}=6.1$ tonnes. In computations we take the distance between the detector and the upstream absorber (the dirt) to be $\Delta^{\mathrm{MV}}=10 \mathrm{~m}$.

The MINER $\nu \mathrm{A}$ detector consists of scintillator strips, which provide 3D information on the tracks. Good particle ID allows one to distinguish the $1 e$ from $1 \gamma$ and $e^{+} e^{-}$ showers using the energy loss $d E / d x$ (along the track or in the first four strips). Three different samples of data were explored: the CCQE $\nu$ interactions and the $\nu e$ scattering data at LE and HE.

(a) e-like events from the $\nu_{e}$ CCQE interactions.-A total number of $3204 e$-like events was observed, while 2931 events were expected [37]. We sum the statistical uncertainty of the observed number of events, $\Delta^{\text {stat }}=56.6$, and $15 \%$ systematic uncertainty, $N^{\text {syst }}=480.7$, quadratically which gives the upper bounds on the new physics contribution:

$$
N_{e}^{\mathrm{MV}}<757(1 \sigma), 1241(2 \sigma), 1725(3 \sigma) .
$$

As the signature selection efficiency, we use the energyaveraged selection efficiency for the electron showers from the $\nu-e$ scattering analysis in Ref. [38]: $\epsilon_{\gamma}^{\mathrm{MV}}=70 \%$. (b) $\gamma$-like events from the $\nu-e$ scattering analysis.-The single EM shower events have been detected in interactions of the LE neutrino flux produced by $3.43 \times 10^{20}$ POTs in Ref. [38]. The $d E / d x$ distribution of the events was constructed (cf. Fig. 3 in Ref. [38]), which allows one to disentangle events produced by electrons and gammas. For $d E / d x>4.5(\mathrm{MeV} / 1.7 \mathrm{~cm}), 171$ photonlike events were observed which practically coincide with the number of expected 170 events. The statistical error $\Delta N^{\text {stat }}=13.1$ and the systematic error $\Delta N^{\text {syst }}=17.1$ (using $10 \%$ error according to Ref. [38]) allow us to get upper bounds on new physics contributions to single-shower events:

$$
N_{\gamma / e e}^{\mathrm{MV}}<23(1 \sigma), 45(2 \sigma), 66(3 \sigma)
$$

A similar analysis has been carried out with the ME data [39], $1.16 \times 10^{21}$ POTs. Following the same procedure as above, $1466 \gamma$ events were observed and 1395 events were expected. We add in quadrature the statistical error $\Delta N^{\text {stat }}=38.3$ and the systematic error $\Delta N^{\text {syst }}=146.6$, which is the $10 \%$ error presented in Ref. [39]. This gives the upper bounds on single-shower events:

$$
N_{\gamma / e e}^{\mathrm{MV}}<223(1 \sigma), 374(2 \sigma), 526(3 \sigma)
$$

Since no photon particle identification cut on the data has been employed, the results can be applied to $\xi=\gamma$ and collimated electron-positron pairs, $\xi=e^{+} e^{-}$. Our statistical analysis shows that constraints on the allowed number of additional photonlike events are the strongest when considering this ME dataset.

We set the probability that a $\xi$ is accepted as a single EM shower to one: $f_{\xi-1 s h}=1$. We account for the cut $E \theta^{2}<$ $0.0032 \mathrm{GeV}$ in MINER $\nu \mathrm{A}$ with an estimated selection efficiency of $10 \%$ that is inferred from SM processes in Fig. 4 in Ref. [38]. Here, $E$ is the shower energy and $\theta$ is the angle between the direction of emitted charged particle(s) that yield a shower and the incoming active neutrino. We found that events surviving the cut on $E \theta^{2}$ would not induce observable hadronic activity in MINER $\nu \mathrm{A}$.

\section{PS191}

The PS191 experiment was sourced by the PS proton beam with energy $19.2 \mathrm{GeV}$ interacting with a beryllium target, and it collected $2 \times 10^{19}$ POTs. The $\nu_{\mu}$ flux at the detector from pion decays was $\phi_{\nu_{\mu}}^{\pi}=2.3 \times$ $10^{-4} \mathrm{~cm}^{-2} \mathrm{POT}^{-1}$. The setup has the parameters $l^{\mathrm{PS}}=$ $128 \mathrm{~m}$ and $l_{p}^{\mathrm{PS}}=49.1 \mathrm{~m}$. The detector was composed of a decay volume and a downstream calorimeter. The decay volume of length $d^{\mathrm{PS}}=12 \mathrm{~m}$ was filled in with flash chambers for tracking and helium bags and, therefore, had negligible mass. The calorimeter consisted of sandwiches made from flash chambers and 3-mm-thick iron plates. Two studies have been performed. 
(a) Two tracks in the decay volume.-Events induced by heavy neutrino decays in the decay volume were searched for in Ref. [40]. These events should have two tracks in the decay volume and an energy deposit in the calorimeter. The vertex of the two tracks can be reconstructed. The criteria was that the reconstructed vertex should be more than $2 \mathrm{~cm}$ away from a flash chamber. Not a single vertex was found; this null result constrains the contribution from heavy neutrinos with decay into $\xi$ that leaves two charged tracks in the flash chambers. The limit on events with two tracks reads [40]

$$
N_{2 \text { tr }}^{\mathrm{PS} \text { obs }}<2.3,95 \% \text { C.L. }
$$

We apply this limit for the final states $\xi=\gamma \gamma$ and $e^{+} e^{-}$with an invariant mass above the threshold $W_{c}^{\mathrm{PS}}=30 \mathrm{MeV}$. This threshold was derived from Ref. [40], where heavy neutrinos with $m_{N} \approx 30 \mathrm{MeV}$ are still subject to constraints. For the signature selection efficiency, we use the signal selection efficiency $\epsilon_{2 \mathrm{tr}}^{\mathrm{PS}}=0.28$ taken from Ref. [40].

(b) Single showers in the calorimeter.-Good granularity of the calorimeter allows one to distinguish the photon showers from the electron showers. In Ref. [41], the electromagnetic showers with energies above $400 \mathrm{MeV}$ were selected to suppress background from $\pi^{0}$ decay. As a proxy for the signal selection efficiency, we use the reconstruction efficiency from Ref. [40]: $\epsilon_{1 s h}^{\mathrm{PS}}=0.7$. Showers can be produced by $\nu_{\mu}$ interactions, in particular, from final states including $\gamma, \pi^{0}$, and $e$, and by hadrons. Hadron misidentification is at most $1 \%$. The subsample with an electron-likelihood selection cut yields an excess of the $e$-like events in the calorimeter:

$$
N_{1 s h}^{\mathrm{PS}, \mathrm{obs}}=23 \pm 8,
$$

that was attributed to neutrino oscillations [41].

\section{NOעA near detector}

The NO $\nu \mathrm{A}$ experiment uses the NuMI neutrino beam sourced by interactions of $120 \mathrm{GeV}$ protons with a graphite target. The parameters of setup are $l^{\mathrm{NOV}}=1000 \mathrm{~m}$, $l_{p}^{\mathrm{NOV}}=675 \mathrm{~m}$, and $14.6 \mathrm{mrad}$ offline detector. The detector is a tracking calorimeter composed of fine-grained cells of liquid scintillator with a total mass of 193 t. Particle identification is based on the topological information from the tracking of particles and uses advanced pattern recognition algorithms.

Single isolated e shower.-The event sample corresponds to $1.66 \times 10^{20}$ POTs. The analysis in Ref. [42] selects neutrino interaction candidates with total energy in the range $1.5-2.7 \mathrm{GeV}$, and the maximal $\nu_{e}$ signal is expected around $2 \mathrm{GeV}$. For the signature selection efficiency, we adopt the signal selection efficiency $\epsilon_{e}^{\mathrm{NOV}}=$ $33 \%$ [42].
The observed event distribution in the calorimetric energy shows good agreement between observed, $N_{e}^{\text {NOVA,obs }}=$ 2573 , and predicted, $N_{e}^{\mathrm{NOVA}, \text { th }}=2385$, numbers of events. Using the statistical uncertainty $\Delta N^{\text {stat }}=50.7$ and the $15 \%$ systematic uncertainty $\Delta N^{\text {syst }}=385.9$, we find bounds on new physics contribution:

$$
N_{e}^{\mathrm{NOV}}<577(1 \sigma), 966(2 \sigma), 1355(3 \sigma) .
$$

\section{NOMAD}

We also considered the NOMAD experiment with $450 \mathrm{GeV}$ protons impinging on a beryllium target, a total POT of $2.2 \times 10^{19}$, a baseline of $620 \mathrm{~m}$, and a detector with length of $3.7 \mathrm{~m}$ and target mass of $3.6 \mathrm{t}$. Among others, the collaboration performed a search for forward photons in Ref. [43] to test the model from Ref. [5]. We found that, in general, NOMAD has less testing power compared to the other detectors; hence, we will not discuss it further in the following.

\section{On discovery potential}

Experiments under consideration are all of the same type: accelerator experiments with near or relatively close detectors. Therefore, it is straightforward to compare their discovery potentials. In various cases, one can simply compare the "strengths" of experiments defined as the product of POTs, efficiencies, and masses of detectors:

$$
\kappa^{i} \equiv(\mathrm{POT})^{i} \times \epsilon^{i} \times M^{i} .
$$

Notice that, for scenarios with decay, the active volume of a detector is relevant and not the mass.

Apart from this product, also other factors are important: the energy of protons and composition of a target which determine multiplicities of secondary particles and, consequently, fluxes of neutrinos. The length of the baseline gives a spread of the neutrino or new particles beams, etc. Therefore, instead of (POT), one can use immediately the neutrino fluxes at detectors:

$$
\kappa_{\nu}^{i} \equiv \phi_{\nu}^{i} \times \epsilon^{i} \times M^{i},
$$

or the fluxes of heavy neutrinos. The MB strength is much higher than the ND one: $\kappa^{\mathrm{MB}} \simeq 2 \times 10^{23}$ tons, while for $\mathrm{ND} 280 \kappa^{\mathrm{ND}}=4 \times 10^{21}$ tons. Using the neutrino fluxes we obtain comparable strengths: $\kappa_{\nu}^{\mathrm{MB}}=5.4 \times 10^{13}$ ton $\mathrm{cm}^{-2}$ and $\kappa_{\nu}^{\mathrm{ND}}=2.1 \times 10^{13}$ ton $\mathrm{cm}^{-2}$, although the MB strength is still 2.5 times larger.

Further contribution to the discovery potential comes from particle ID. Experiments with better ID gain, since a smaller subset of events can be selected, and, therefore, stronger bounds on new physics contributions can be obtained. This can be accounted by the ratio of the strength over the upper bound on the observed number of events: $\kappa_{\nu}^{i} / N^{i}$. Thus, MiniBooNE has observed 638 one-shower 
TABLE II. Parameters that enter in the analysis. For T2K280, we list two numbers for detector mass and its length. This is because we include the possibility of the upscattering in the P0D with $1 \mathrm{~m}$ distance from TPC-FGD system.

\begin{tabular}{|c|c|c|c|c|c|c|}
\hline Experiment & MiniBooNE & $\mathrm{T} 2 \mathrm{~K}$ & NOMAD & PS191 & MINER $\nu \mathrm{A}$ & $\mathrm{NO} \nu \mathrm{A}$ \\
\hline Area $\left(\mathrm{m}^{2}\right)$ & $36 \pi$ & 3.47 & 6.76 & 18 & 1.71 & 12.39 \\
\hline$d(\mathrm{~m})$ & $2 / 3 \cdot 12$ & $d_{1}=1, d_{2}=0.9$ & 3.7 & 3.55 & 3 & 8 \\
\hline$l_{p}(\mathrm{~m})$ & 50 & 94 & 290 & 49.1 & 675 & 675 \\
\hline POT $(\nu+\bar{\nu}$ mode $)$ & $3 \times 10^{21}$ & $1.821 \times 10^{21}$ & $2.2 \times 10^{19}$ & $0.86 \times 10^{19}$ & $3.43 \times 10^{20}$ & $1.66 \times \cdot 10^{20}$ \\
\hline$M$ (tonnes) & 818 & $m_{P 0 D}=15.8, m=1.1$ & 112 & 20 & 6.1 & 300 \\
\hline$\nu$ energy range $(\mathrm{GeV})$ & {$[0.1-5]$} & {$[0.1-10]$} & {$[5-200]$} & {$[0.1,5]$} & {$[0.1-20]$} & {$[0.1-20]$} \\
\hline
\end{tabular}

events, while the ND280 upper bound is about 150 . That is, ND280 gains a factor of 3, and its discovery potential becomes even slightly higher than the one of MiniBooNE. Further improvements can be related to the specific scenario and geometry of experiment. Thus, ND280 can gain in the decay scenarios because of a smaller baseline. This is precisely the origin of upturns (see below) where the bound becomes stronger. To a large extent, this enhancement is artificial and related to geometric suppression of the number of the MB events. In upscattering scenarios, sizes of detectors become important. Similarly, one can consider discovery potential of other experiments and searches.

For convenience, we summarize relevant parameters of the experiments under discussion in Table II. We provide the salient information on analyses of data, signatures, and the upper bounds on the number of new physics events in Table III. These bounds (see the fourth row) will be confronted with theoretical predictions in Sec. V.

\section{E. Uncertainties}

Let us briefly discuss uncertainties in the predictions of the number of events $N^{i}$ according to Eq. (18). The uncertainty from the cross section evaluations were discussed in Sec. IV B.

(i) The uncertainty of the number of MiniBooNE excess event are given by the collaboration: $\delta_{N_{\text {sh } h \text {,exp }}^{\mathrm{MB}}} \approx 0.2$.

(ii) The predictions use input parameters, such as POT, baseline, and detector properties (mass, density, geometry, etc.). They are extremely well controlled experimentally, and, therefore, we neglect the associated uncertainties. A relevant source of uncertainty is the $\phi_{\pi}^{0}$ flux estimated as $\mathcal{O}(10 \%)$.

(iii) For the cases of upscattering in dirt, we parametrize the distance between upstream dirt and the detector with a single quantity $\Delta$, ignoring the 3D geometry of a setup. In principle, upscattering in the upstream dirt has to be taken into account via a Monte Carlo simulation, which covers the full geometry of the detector hall and the experiment, as well as the model-specific scattering cross section. This is clearly beyond the scope of our work. We estimated a relative error due to this simplification by varying $\Delta$ between 1 and $30 \mathrm{~m}$. We find that variation affects the predictions of $N^{i}$ only for $c \tau^{0} \gtrsim 1 \mathrm{~m}$ and $\delta_{\Delta}=0.4$. This is comparable to the uncertainty in the density of dirt: Its variation between 2 and $4 \mathrm{~g} / \mathrm{cm}^{3}$ leads to $\delta_{n_{b}}=0.3$.

Since the above mentioned uncertainties are multiplicative factors in our prediction, we combine them in quadrature. Assuming a relative uncertainty for all fluxes to be 0.2 , we have for the mixing decay scenarios $M_{N} D_{\xi}$ and $M_{N} D_{\nu} U_{e}$

$$
\delta_{M}^{i}=\delta_{N} \oplus \delta_{\phi_{0}}^{\mathrm{MB}} \oplus \delta_{\phi_{0}}^{i} \simeq 0.35
$$

For the upscattering scenarios $U_{N} D_{\xi}$ and $U_{N} D_{B} D_{\xi}$, we have to add uncertainties due to the parameters of dirt (distance to a detector and density) and cross sections. For

TABLE III. Summary of considered experimental searches, signatures, and the upper bounds that will be used to constrain scenarios explaining MiniBooNE.

\begin{tabular}{|c|c|c|c|c|}
\hline Experiment & Analysis & Signature & Upper limit $1 \sigma / 3 \sigma$ & Reference \\
\hline \multirow[t]{3}{*}{ T2K ND280 } & Heavy neutrino decays & $e^{+} e^{-}$ & $20 / 49$ & [36] \\
\hline & CCQE electrons & $e^{-}\left(e^{+}\right)$ & $17 / 261$ & [23] \\
\hline & CCQE electrons & Single $\gamma$ & $58 / 305$ & [23] \\
\hline $\mathrm{NO} \nu \mathrm{A}$ & CCQE electrons & $e^{-}$ & $577 / 1355$ & [42] \\
\hline \multirow[t]{3}{*}{$\operatorname{MINER} \nu \mathrm{A}$} & CCQE electrons & $e^{-}\left(e^{+}\right)$ & $757 / 1725$ & [37] \\
\hline & Neutrino electron scattering & EM shower, or $\gamma, e e$ & $23 / 66$ & [38] \\
\hline & Neutrino electron scattering & EM shower, or $\gamma, e e$ & $223 / 526$ & [39] \\
\hline NOMAD & Single-photon search & Single $\gamma$ & $18 / 50$ & [43] \\
\hline \multirow{2}{*}{ PS191 } & Heavy neutrino decays & Displaced vertex & $1.84 / 6.61$ & [40] \\
\hline & Neutrino oscillation & Electronlike events & $23 \pm 8$ & [41] \\
\hline
\end{tabular}


the latter, we use $\delta_{\sigma}^{\mathrm{MB}-i}=0.2$ according to Sec. IV B. As a result,

$$
\delta_{U}^{i}=\delta_{M} \oplus \delta_{n_{b}}^{\mathrm{MB}} \oplus \delta_{n_{b}}^{i} \oplus \delta_{\sigma}^{\mathrm{MB}-i} \oplus \delta_{\Delta} \simeq \begin{cases}0.55 & c \tau^{0}<1 \mathrm{~m} \\ 0.68 & c \tau^{0} \geq 1 \mathrm{~m}\end{cases}
$$

(iv) Further sources of uncertainties are the detection efficiency and the selection efficiency. Experimental collaborations evaluate both of these efficiencies through Monte Carlo simulations for specific models. This is, however, for the moment done only for SM processes; in other words, new physics interactions have not yet been properly implemented in generators such as GENIE. Our approach is, therefore, for a particular process, to adopt the efficiency from a similar (identical signature, for instance, single shower in the final state) SM process quoted by experimental collaborations. We expect this to yield a very good proxy for the new physics process efficiencies. We list all employed efficiencies in Sec. IV, and, if experimental collaboration moves in the direction of considering such specific processes in the years to come, our results could be straightforwardly rescaled with the new values that we do not expect to significantly depart from those employed in this paper.

\section{TESTS OF SCENARIOS}

The bounds obtained in Sec. IV apply to the final states of different scenarios. Therefore, two different scenarios with the same final EM state have the same tests. The difference is in implications, that is, in the level of restrictions of scenarios. Furthermore, due to misidentification, any signature $s^{i}$ provides bounds on all possible final states $\xi$ and, consequently, scenarios. We call the direct test when the EM component of final state, $\xi$, coincides with signature: e.g., $e-e$ shower, etc. The indirect tests require misidentification. The most stringent bounds (the best tests) are provided by the direct tests, since misidentification brings certain smallness.

Several different experiments measure the same type of events (signatures), but the best bound is given by experiment which has the highest strength. The latter allow us to identify the relevant experimental results for each scenario.

Recall that, according to Eq. (18), the predictions of numbers of events for all detectors are normalized to the MiniBooNE excess, i.e., to the number of one-shower events, $N_{\xi-1 s h}^{\mathrm{MB}}$, and the latter is proportional to $f_{\xi-1 s h}$.

\section{A. Mixing-decay scenario, $M_{N} D_{\xi}$}

This is the simplest scenario with only two new physics interaction points: the production point of $N$ via mixing and the $N$-decay point (see Fig. 1 ). $N$ with mass $m_{N} \leq 10 \mathrm{MeV}$ is produced in the $\pi$ decays in the decay pipe, and it decays along the beam line.

The typical dependence of the number of events on $c \tau^{0}$ (see Sec. III B) has the exponential upturn and constant asymptotics at $c \tau^{0} \rightarrow \infty$ (see Figs. 7 and 8 ). The upturn point is determined by the baseline and typical energy of the MiniBooNE experiment [1]. In our approximation of the $E_{N}$-independent signature, factors such as behavior are the same for all possible final states $\xi$.

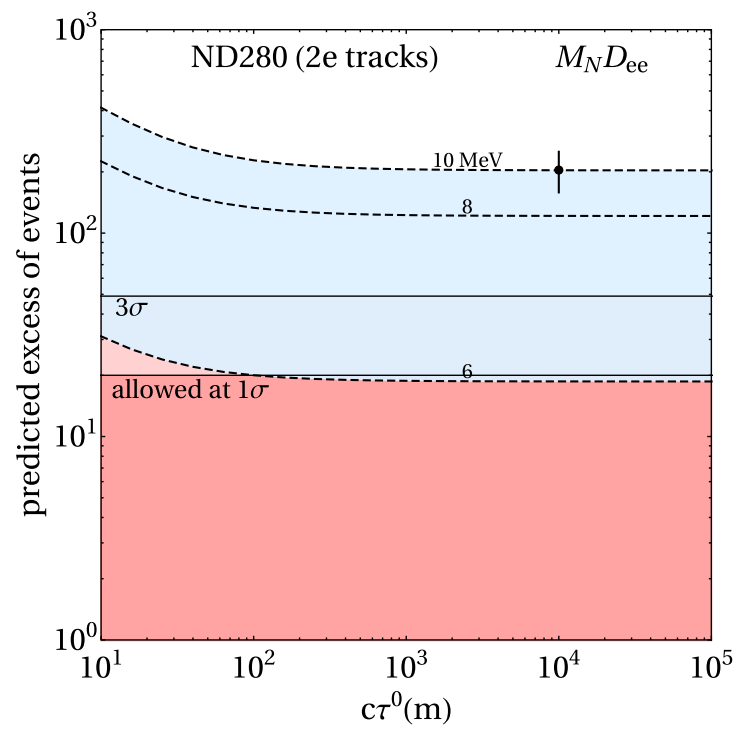

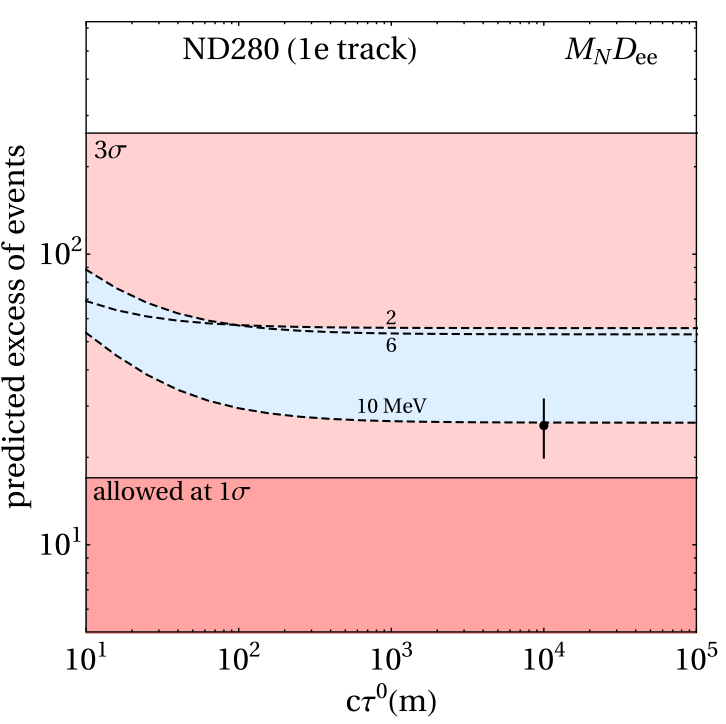

FIG. 7. Tests of the mixing-decay into $e^{+} e^{-}$scenario $M_{N} D_{e e}$ at ND280. Left panel: number of expected $2 e$-shower events produced by the $e^{+} e^{-}$pair as a function of $c \tau^{0}$ for different values of $m_{N}$ (numbers at the curves in $\mathrm{MeV}$ ). The point with the error bar indicates the uncertainty of the prediction from the MiniBooNE-observed event rate. Borders of shadowed regions show the $1 \sigma$ and $3 \sigma$ experimental upper bounds on these numbers. Right panel: the same as in the left panel but for the $1 e$-shower events at ND280. 

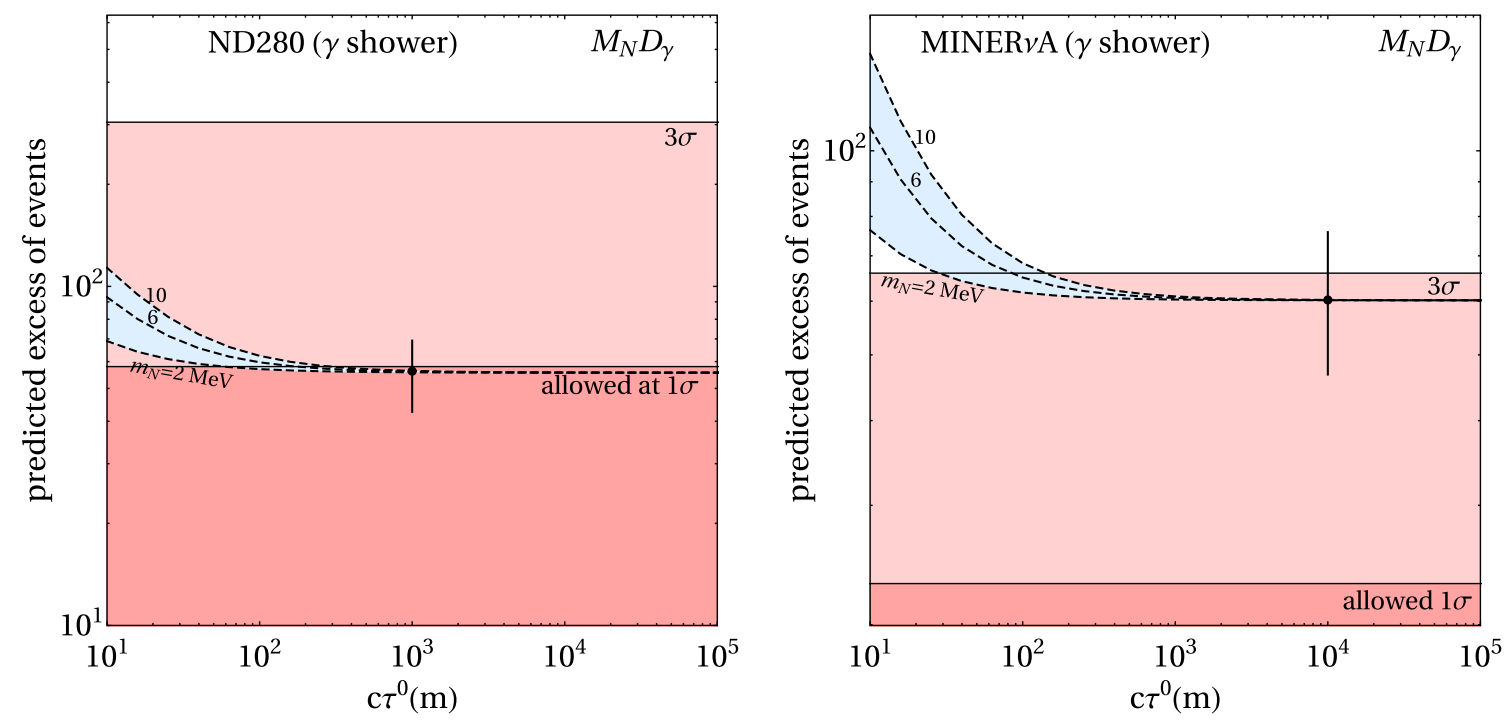

FIG. 8. Tests of the mixing-decay into $\gamma$ scenario, $M_{N} D_{\gamma}$. The number of expected $\gamma$-shower events is shown as a function of $c \tau^{0}$ for different values of $m_{N}$ (numbers at the curves in $\mathrm{MeV}$ ). Borders of shadowed regions show the $1 \sigma$ and $3 \sigma$ experimental upper bounds on these numbers. The point with the error bar indicates the uncertainty of the prediction from the MiniBooNE-observed event rate. Left panel: ND280. Right panel: MINER $\nu$ A.

The absolute value of the excess of events in a given experiment is determined by the product (36). The final states produced in the $N$ decay are $\xi=\gamma$ (radiative decay) and $\xi=e^{+} e^{-}$(three-body decay). Also, the $2 \gamma$ final state can be explored, but $\xi=e$ is not possible. Let us consider $\xi=e^{+} e^{-}$and $\xi=\gamma$ in more detail.

\section{1. $\xi=e^{+} e^{-}: M_{N} D_{e e}$ scenario}

The $N_{e e-2 s h}^{\mathrm{ND}}$ result (88) provides the direct test and, therefore, gives the strongest bound. Bounds from other data rely on the misidentification of $e^{+} e^{-}$showers with $e$ or $\gamma$ showers and require small invariant mass of the $e^{+} e^{-}$pair, $W_{e e}$. In this scenario, an angular selection cut of $\cos \theta \geq$ 0.99 is well satisfied, and, therefore, the selection efficiency is close to $100 \%$.

(a) For the invariant mass of the pair $W_{e e}>W_{c}=$ $5 \mathrm{MeV}$, the electron and positron are resolved in ND280, and, therefore, the bound on $2 e$-shower events $N_{e e-2 s h}^{\mathrm{ND}}(88)$ can be used. In Fig. 7 (left panel), we show the dependence of $N_{e e-2 s h}^{\mathrm{ND}}$ on $c \tau^{0}$ for three values of mass $m_{N}$, allowed by timing restriction (see Sec. IIC and Ref. [2]). In our computations, we used the expression (18) for $N_{e e-2 s h}^{\mathrm{ND}}$ with parameters of the experimental setup given in Table II and $f_{e e-2 s h}$ found with Eq. (86). For the $N$ flux at $m_{N} \lesssim 10 \mathrm{MeV}$, we use the active neutrino flux reduced by the mixing parameter $\left|U_{\mu N}\right|^{2}$ as a proxy.

Figure 7 shows very strong dependence of the expected number of events on $m_{N}$ which comes mainly from the signature factors. Indeed, $N_{e e-2 s h}^{\mathrm{ND}} \propto f_{e e-2 s h}^{\mathrm{ND}} / f_{e e-1 s h}^{\mathrm{MB}}$. In MiniBooNE, with $W_{c}=30 \mathrm{MeV}$, the $e^{+} e^{-}$pairs are not resolved: $W_{e e}<m_{N}<W_{c}$, so that $f_{e e-1 s h}^{\mathrm{MB}}=1$. In ND280, the values of mass $m_{N}$ are close to the threshold, and, therefore, $f_{e e-2 s h}^{\mathrm{ND}}$ increases strongly with $m_{N}$.

According to the figure, the $M_{N} D_{e e}$ scenario with $m_{N}>$ $7 \mathrm{MeV}$ is excluded. The bound relaxes with a decrease of $m_{N}$, being below $\sim 1 \sigma$ for $m_{N}<7 \mathrm{MeV}$.

(b) For $W_{e e}<5 \mathrm{MeV}$, the $e^{+} e^{-}$pairs show up in ND280 as $1 s h$ events. Their number can be restricted by results of studies of the $e$ showers produced by the $\nu_{e}$ CCQE at ND280, as well as at PS191, NOMAD, and MINER $\nu$ A. Notice that this is an indirect test which relies on misidentification.

In Fig. 7 (right panel), we show the expected number of one-shower events at ND280 produced by the $e^{+} e^{-}$pairs. The dependence of $N_{e e-1 s h}^{\mathrm{ND}}$ on $m_{N}$ is strong but opposite to that for the two-shower events: $N_{e e-1 s h}^{\mathrm{ND}}$ decreases with an increase of $m_{N}$, again, due to signature factor $f_{e e-1 s h}^{\mathrm{ND}}$. According to Eq. (86), for $m_{N}$ above the threshold, $f_{e e-1 s h}^{\mathrm{ND}} \propto W_{c}^{2} / m_{N}^{2}$. (This reflects the fact that probability of the three-body $N$ decay with invariant mass of the pair $W_{e e}<W_{c}$ decreases.) The opposite dependence of number of events on $m_{N}$ in $1 s h$ and $2 s h$ cases can be also inferred from the sum rule: $f_{e e-2 s h}^{\mathrm{ND}}=1-f_{e e-1 s h}^{\mathrm{ND}}$.

We confront the predictions with the bound (90). According to Fig. 7 (right panel), the $M_{N} D_{e e}$ scenario with $m_{N}<6 \mathrm{MeV}$ is disfavored at about $2 \sigma$ level in the whole range of $c \tau^{0}$. The bound weakens with the increase of $m_{N}$.

For small $W_{e e}$, the final $e^{+} e^{-}$state can also be misidentified with a $\gamma$ shower. In such a case, the bounds on $1 \gamma$-shower searches of new physics by NOMAD, 
ND280, PS191, and MINER $\nu$ A can be applied [see, for instance, Eqs. (89) and (93)].

\section{2. $\xi=\gamma, M_{N} D_{\gamma}$ scenario}

The direct tests of this scenario are provided by the $1 \gamma$ shower searches of new physics at ND280, MINER $\nu$ A, and NOMAD. In Fig. 8, we present results for ND280 (left) and MINER $\nu$ A (right). NOMAD gives much weaker bounds than ND280 and MINER $\nu$ A. In our computations, we used $f_{1 \gamma}=1$ and the values of $\epsilon$ from Table II (see also Sec. IV). According to this figure, the predicted number of $1 \gamma$ events is at the level of the $1 \sigma$ upper bound from ND280; see Eq. (89). Future ND280 data may improve the bound. MINER $\nu \mathrm{A}$ gives a much stronger restriction; see Eq. (93). For $c \tau^{0}>10^{2} \mathrm{~m}$, the prediction is at $3 \sigma$ exclusion, and at $c \tau^{0}<10^{2} \mathrm{~m}$ the bound becomes stronger than $3 \sigma$, especially for larger values of $m_{N}$.

The model with $c \tau^{0} \gtrsim 10^{3} \mathrm{~m}$ and $m_{N} \sim 250 \mathrm{MeV}$ which fits this scenario (but with much larger masses of $N$ ) was proposed in Ref. [6]. It is excluded by timing constraints and independently disfavored by our consideration.

The bounds obtained here can be applied to the mixingdouble-decay scenario $M_{N} D_{B} D_{\xi}$ considered in Sec. III F. In the limits $\lambda_{B} \ll \lambda_{N}$ and $\lambda_{B} \gg \lambda_{N}$, they can be applied immediately. In the case $\lambda_{B} \sim \lambda_{N}$, the predicted number of events should be corrected by factor (78) which is about 0.4 for ND280. For other possibilities, we can introduce scaling: $\lambda_{B}=\alpha \lambda_{N}$ and $m_{B}=\beta m_{N}$, where $\alpha$ and $\beta$ are constants, and present results in the same way as for the two-parameter scenarios, namely, as the number of events as a function of $c \tau_{N}^{0}$ for different values of $m_{N}$. Model [18] fits this scenario with $\lambda_{N} \rightarrow 0$ (or the $U_{N} D_{\xi}$ scenario with $N$ substituted by $B$ ).

\section{B. Upscattering-decay scenario, $\boldsymbol{U}_{N} \boldsymbol{D}_{\boldsymbol{\xi}}$}

Recall that here $N$ is produced by the $\nu_{\mu}$ upscattering in a detector as well as in matter between a decay pipe and a detector. In turn, $N$ decays in the detector (see Sec. III C). This scenario has final states $\xi$ and signatures similar to those of $M_{N} D_{\xi}$, since in both cases the final state is produced in the $N$ decay. The difference is in the geometry of the $N$-production part and, consequently, in the $c \tau^{0}$ dependence, as well as in the larger allowed values of $N$ mass: $m_{N} \gtrsim 100 \mathrm{MeV}$. Timing constraints are much weaker in this scenario with respect to $M_{N} D_{\xi}$.

According to Sec. III C, the contribution to the number of events from the $\nu_{\mu}$ upscattering in the detector has a smoothed steplike dependence on $c \tau^{0}$ with a transition region between the two asymptotics at $D^{i}<c \tau^{0}<D^{\mathrm{MB}}$, where $D^{i} \equiv$ $d_{i} m_{N} / E_{N}$ is the reduced size of a detector. The contribution from the $\nu_{\mu}$ upscattering in outer matter is negligible at small $c \tau^{0}$ and it increases, first linearly, and then reaches its maximum at $D^{i}<c \tau^{0}$ followed by a decrease toward a constant value in the asymptotics. The sum of the two contributions produces a "bumpy" form in the transition region (see Fig. 9 below). The substantial difference from the $M_{N} D_{\xi}$ scenario in terms of tests and relevance of experimental bounds is related to the masses of $m_{N}$, which affects the signature factors $f$. The latter can suppress or enhance

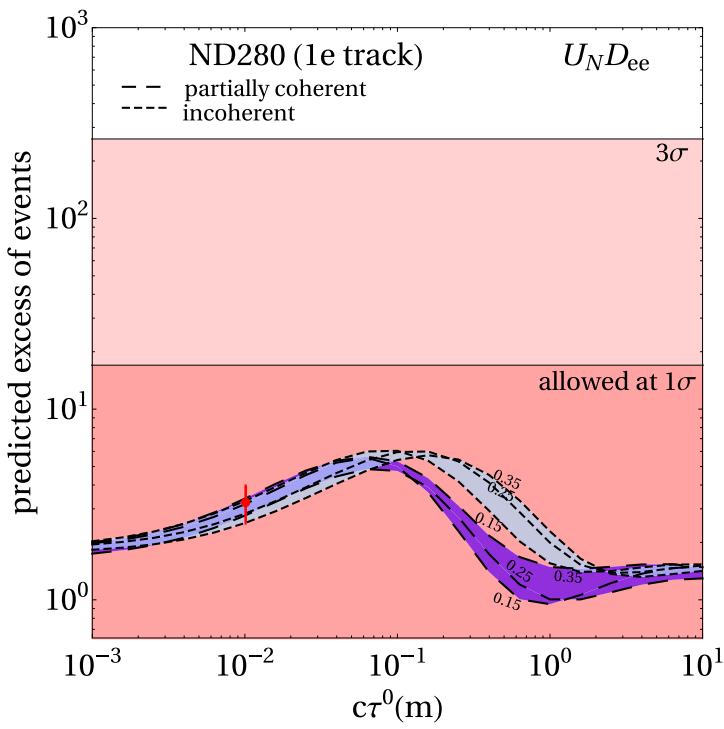

FIG. 9. Tests of the upscattering-decay into $e^{+} e^{-}$scenario, $U_{N} D_{e e}$ at ND280. Left panel: the number of expected $2 e$-track events produced by the $e^{+} e^{-}$pairs as a function of $c \tau^{0}$ for different values of $m_{N}$ (numbers at the curves in GeV). The point with the error bar indicates the uncertainty of the prediction from the MiniBooNE-observed event rate. Two sets of lines correspond to contributions computed with partially coherent and incoherent cross sections. The horizontal lines show the $1 \sigma$ and $3 \sigma$ experimental upper bounds. Right panel: the same as in the left panel, but for the $1 e$-track events at ND280. 
expected numbers of events. The final states $\xi$ can be $e^{+} e^{-}$ and $\gamma$, and we will consider them in order.

\section{1. $\xi=e^{+} e^{-}-U_{N} D_{e e}$ scenario}

ND280 data on $e^{+} e^{-}$pairs provide the direct test of this scenario. Because of the large mass of $N, m_{N} \gg$ $W_{c}^{\mathrm{ND}}=5 \mathrm{MeV}$, the signature factor $f_{e e-2 e s h}$ is close to 1 . We evaluated the efficiency of the angular selection cut $\cos \theta>0.99$ for $m_{N}$ masses of 150,250 , and $350 \mathrm{MeV}$ (indicated in the figures) and gauge boson masses corresponding to the benchmark points or Refs. [10] (partially coherent) and [7] (incoherent). We found that for incoherent (partially coherent) scattering roughly $10 \%$ (40\%) of the signal events pass this selection cut.

Furthermore, we found that this angular cut corresponds to the hadronic recoil momenta below the detection threshold, which is $\sim 400 \mathrm{MeV}$ in ND280 [44]. This means that incoherent scattering will not receive further efficiency reductions from veto on events related to the absence of hadron activity.

In the left panel in Fig. 9, we show the predicted number of $2 e$-track events, $N_{e e-2 e t r}^{\mathrm{ND}}$, as a function of $c \tau^{0}$. The theoretical value $N_{2 e-2 s h}^{\mathrm{ND}}$ has been computed using Eqs. (18) and (47). The $N$ flux at the detector was found using Ref. [35]. The bump in the prediction at $c \tau^{0} \simeq 0.1 \mathrm{~m}$ is due to the contribution from $\nu_{\mu}$ upscattering in the pion detector (P0D) in addition to scattering in the TPC + FGD system, and we consider detection of events in the latter only. The bump is significant, since P0D has a larger mass than TPC-FGD. The surrounding dirt with length $b=$ $140 \mathrm{~m}$ has also been taken into account.

The predicted number of events strongly depends on $m_{N}$. This dependence follows from the MB signature factor $f_{e e-1 s h}^{\mathrm{MB}}$ which appears in the expression for $N_{e e-1 s h}^{\mathrm{MB}}$ in the denominator of Eq. (18). From Eq. (86), we have

$$
f_{e e-1 e}^{\mathrm{MB}} \sim \frac{2\left(W_{c}^{\mathrm{MB}}\right)^{2}}{m_{N}^{2}},
$$

while in the numerator $f_{e e-2 e t r}^{\mathrm{ND}} \approx 1$. Consequently, $N_{e e-2 e t r}^{\mathrm{ND}, o b s} \propto m_{N}^{2}$. Let us underline that this dependence on $m_{N}$ comes from the theoretical number of events at MiniBooNE: With an increase of $m_{N}$, the decrease of $f_{e e-1 s h}^{\mathrm{MB}}(100)$ should be compensated by increasing other factors in $N_{e e-1 s h}^{\mathrm{MB}}$ (e.g., coupling constants) which are also present in the expression for $N_{e e-2 e s h}^{\mathrm{ND}}$.

In Fig. 9, two sets of lines correspond to the partially coherent $N$ production on nuclei realized for light mediators $(\sim 30 \mathrm{MeV})$ and to the incoherent $N$ production due to heavy $(>1 \mathrm{GeV})$ mediators (see the corresponding discussion in Sec. IV). The difference between usage of these two types of cross sections is not large, since the same type of cross section is used in the numerator and denominator of Eq. (18). The mild differences appear in the intermediate region of $c \tau^{0}$ where P0D and dirt also contribute.

According to the left panel in Fig. 9, the experimental bound (88) excludes the scenario for $c \tau^{0} \gtrsim 10^{-2} \mathrm{~m}$ and $m_{N}>50 \mathrm{MeV}$ at more than $3 \sigma$ confidence level. For smaller values of $c \tau^{0}$ this exclusion weakens exponentially, because $N$ produced in the FGD would decay already within FGD and that would be vetoed. The model in Ref. [7] matches this scenario with $m_{N}=110 \mathrm{MeV}$ and $c \tau^{0} \gtrsim 1 \mathrm{~m}$, where $N$ is produced incoherently, since the mediator mass for the benchmark point is $1.25 \mathrm{GeV}$. Such model is excluded by the $2 e$-track ND280 data. (See [45] for the independent test of this model in Icecube).

As a representative of an indirect test for this scenario, we use the $1 e$-track events studied at ND280. The right panel in Fig. 9 shows the predicted excess of one-track events induced by the $e^{+} e^{-}$pairs. These events require very low $W_{e e}$ and the $e e-1 s h$ misidentification. The predicted number of excess events has dependence on $c \tau^{0}$ similar to that in the left panel. Since the signature factors for both ND280 and MiniBooNE have the same $1 / m_{N}^{2}$ dependence, there is no signature factor enhancement and dependence of predictions on $m_{N}$ is much weaker than in the $\xi=e^{+} e^{-}$ case. The predicted excess of events is below $1 \sigma$ limits from Eq. (90).

The direct test of the $U_{N} D_{e e}$ scenario is given by the bound on the two-track events from the PS191 experiment (95). In the left panel in Fig. 10, we show the dependence of $N_{e e-2 t r}^{\mathrm{PS}}$ on $c \tau^{0}$. For PS191 we did not include the dirt contribution. Hence, in both panels one finds the expected smoothed step form of the dependence. The dependence on $m_{N}$ has the same origin as in Fig. 9. The total number of expected events is, however, much smaller than in ND280 due to low strength $\kappa_{\nu}$ for PS191, in particular, due to a low number of POTs (see Table II). The strong bound (more than $3 \sigma$ ) on this scenario appears for large values of masses, $m_{N}>0.25 \mathrm{GeV}$, and short decay lengths: $c \tau^{0}<(0.1-1) \mathrm{m}$.

In the right panel in Fig. 10, we show the prediction for the number of $1 s h$ events originated from the $e^{+} e^{-}$pairs. Misidentification $e^{+} e^{-}-1$ sh requires the low threshold $W_{e e}<W_{c}^{\mathrm{PS}}=30 \mathrm{MeV}$. According to Fig. 10, the $U_{N} D_{e e}$ scenario could explain the observed excess of events at PS191. However, the required values of parameters are already excluded at more than $3 \sigma$ by two-track events at ND280 (see Fig. 9).

\section{2. $\xi=\gamma: U_{N} D_{\gamma}$ scenario}

It can be directly tested at several detectors and, in particular, at MINER $\nu \mathrm{A}$ and ND280.

In the left panel in Fig. 11, the number of isolated $\gamma$ events in MINER $\nu \mathrm{A} N_{\gamma-\gamma s h}^{\mathrm{MV}}$ is shown as a function of $c \tau^{0}$. Both contributions from upscattering in the detector and in the dirt are included; the latter induces a bump at $c \tau^{0}=(1-5) \mathrm{m}$ 

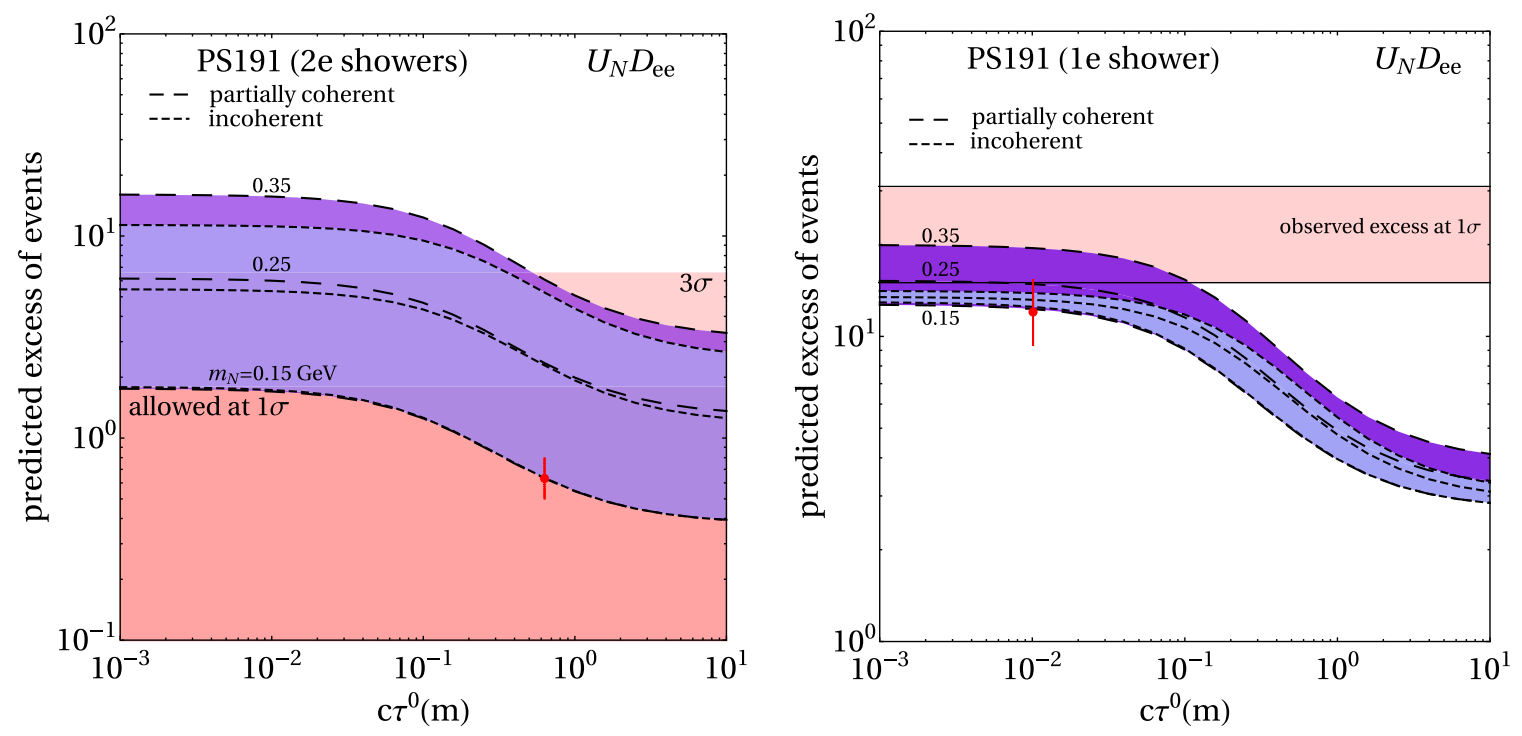

FIG. 10. The same as in Fig. 9 but at PS191.

depending on the value of $m_{N}$ (if there was no dirt effect included, the shape would qualitatively resemble Fig. 10). In both MINER $\nu \mathrm{A}$ and MiniBooNE, the signature factors for this channel are close to 1 and the strong dependence of $N_{\gamma-\gamma s h}^{\mathrm{MV}}$ on $m_{N}$ follows from the coherent cross section: With the increase of $m_{N}$, the cross section for partially coherent scattering drops strongly around the typical MiniBooNE energy $E_{N}^{\mathrm{MB}} \sim 0.8 \mathrm{GeV}$, while for MINER $\nu \mathrm{A}$ with $E_{N}^{\mathrm{MV}} \sim$ $5 \mathrm{GeV}$ the decrease is much weaker:

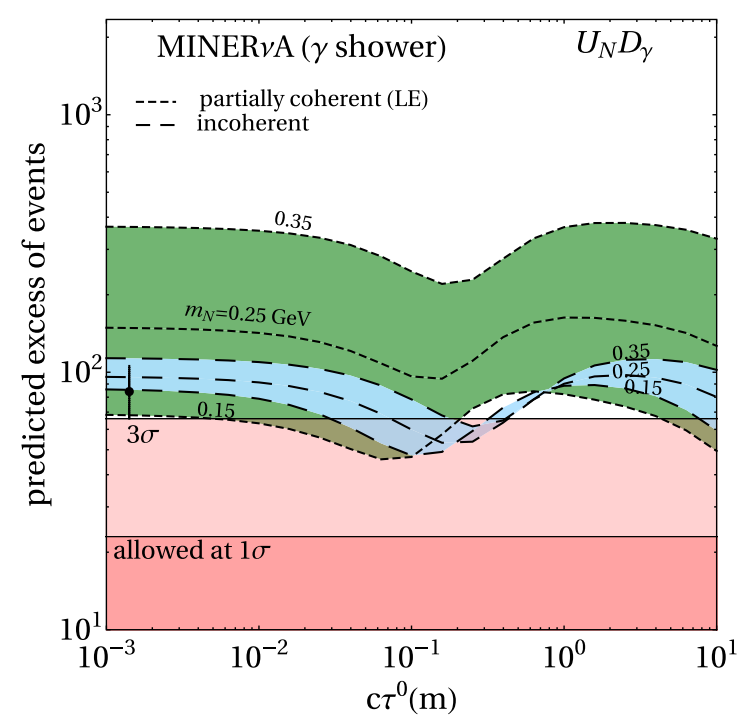

$$
N_{\gamma-\gamma s h}^{\mathrm{MV}} \propto \frac{\sigma^{\mathrm{coh}}\left(E_{N}^{\mathrm{MV}}, m_{N}\right)}{\sigma^{\mathrm{coh}}\left(E_{N}^{\mathrm{MB}}, m_{N}\right)} .
$$

As a result, $N_{\gamma-\gamma s h}^{\mathrm{MV}}$ increases with $m_{N}$. In the case of incoherent $N$ production, the dependence of the cross section on $m_{N}$ is weak.

According to the left panel in Fig. 11, the experimental result (93) excludes the present scenario in the whole range

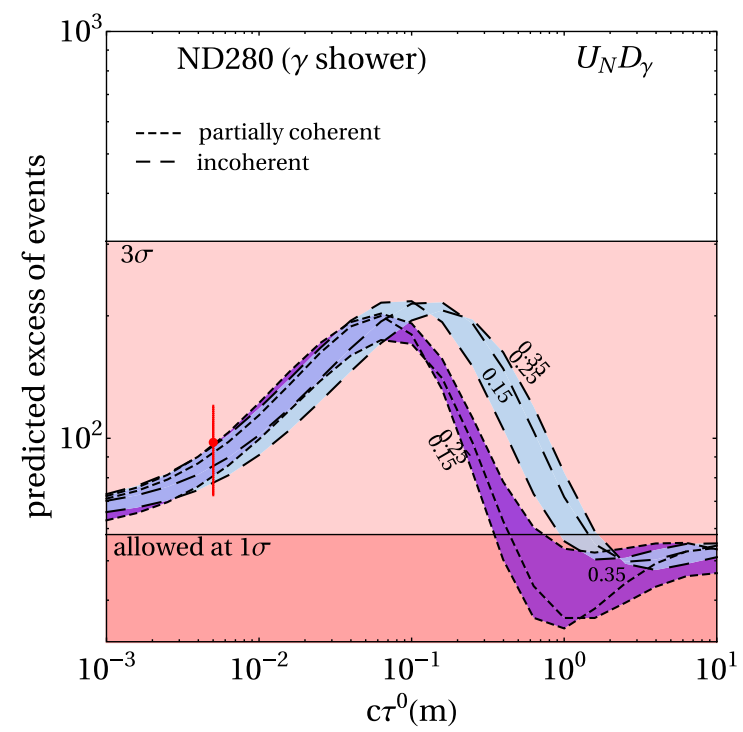

FIG. 11. Direct tests of (bounds on) the upscattering-decay into $\gamma$ scenario $U_{N} D_{\gamma}$ by different experiments. The number of expected $\gamma$-shower events as a function of $c \tau^{0}$ for different values of $m_{N}$ (numbers at the curves in $\mathrm{GeV}$ ) is shown. Horizontal lines show the $1 \sigma$ and $3 \sigma$ experimental upper bounds on these numbers. The point with the error bar indicates the uncertainty of the prediction from the MiniBooNE-observed event rate. Two sets of lines correspond to contributions computed with partially coherent and incoherent cross sections. The left panel is for MINER $\nu \mathrm{A}$, while the right panel corresponds to ND280. 

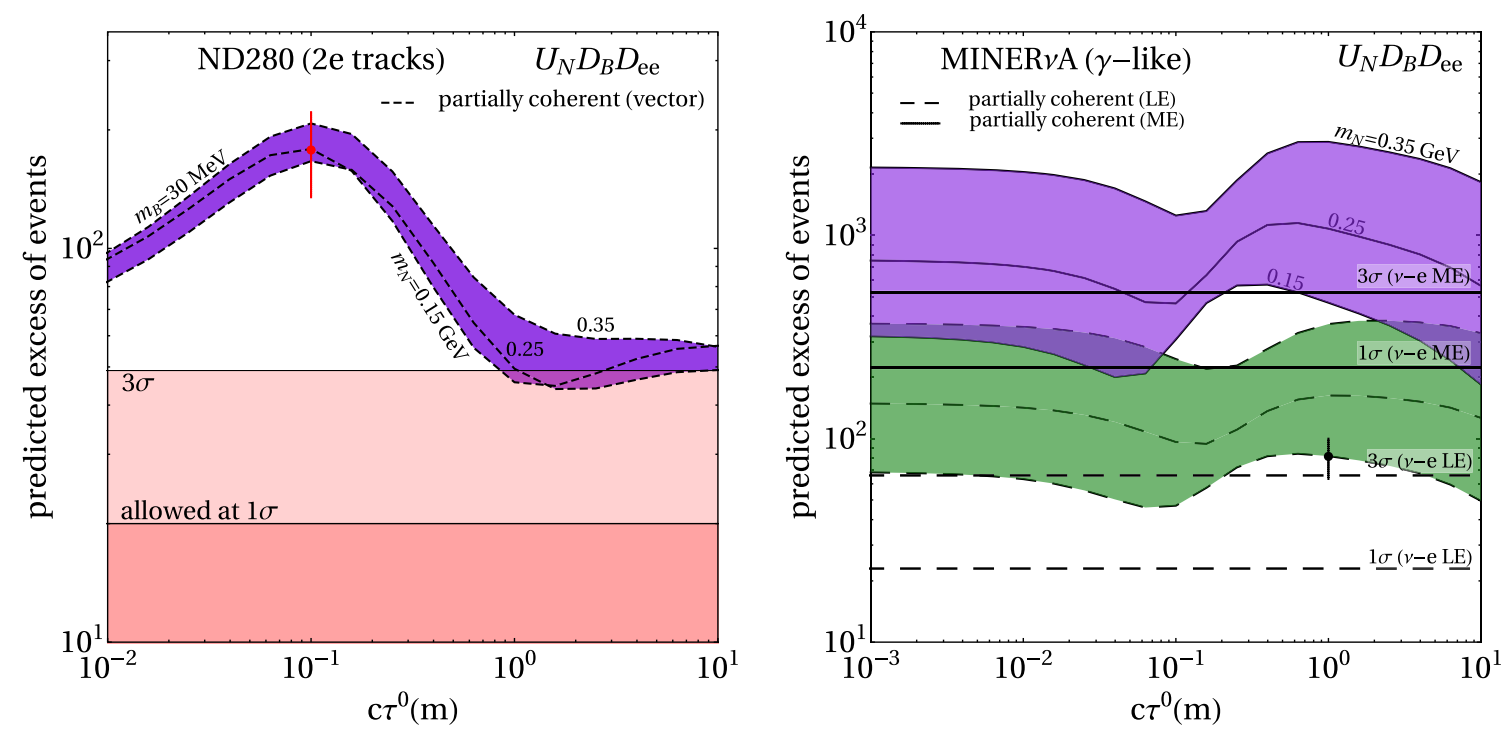

FIG. 12. Tests of the upscattering-double-decay into $e^{+} e^{-}$scenario, $U_{N} D_{B} D_{e e}$ at ND280 (left) and MINER $\nu \mathrm{A}$ (right). Left panel: number of expected $2 e$-track events produced by the $e^{+} e^{-}$pair at ND280 as a function of $c \tau^{0}$ for different values of $m_{N}$ (numbers at the curves in $\mathrm{GeV})$. We take $m_{B}=30 \mathrm{MeV}$. The horizontal lines show the $1 \sigma$ and $3 \sigma$ experimental upper bounds on the $2 e$-track events. The point with the error bar indicates the uncertainty of the prediction from the MiniBooNE-observed event rate. Right panel: number of expected $\gamma$-like shower events at MINER $\nu \mathrm{A}$ as a function of $c \tau^{0}$ for different values of $m_{N}$ (numbers at the curves in GeV). We take $m_{B}=30 \mathrm{MeV}$. Two sets of lines correspond to the contribution of the ME and LE samples of events. A partially coherent cross section was used.

of $c \tau^{0}$ and for $m_{N}>0.1 \mathrm{GeV}$ at the $\sim 3 \sigma$ level. The model [5] fits this scenario with $c \tau^{0}=0.1 \mathrm{~m}$ and $m_{N} \sim 0.5 \mathrm{GeV}$, and it is clearly excluded by MINER $\nu \mathrm{A}$ data.

In the right panel in Fig. 11, we show the excess of single- $\gamma$ events at ND280. The dependence on $c \tau_{0}$ has the typical bump due to contribution from the $N$ production in P0D. The dependence of the excess on $m_{N}$ is weak, since now $E_{N}^{\mathrm{MB}} \approx E_{N}^{\mathrm{ND}}$. The scenario is disfavored at the $(1-2) \sigma$ level, but the bound can be significantly improved in the future with larger datasets.

\section{Upscattering-double-decay scenario, $U_{N} D_{B} D_{\xi}$}

In this scenario (see Fig. 5), $N$ produced via the $\nu_{\mu}$ upscattering in a detector and surrounding materials decays into on-shell boson $N \rightarrow B+\nu$, which, in turn, decays as $B \rightarrow e^{+} e^{-}$. Alternatively, $B$ can undergo a radiative decay $B \rightarrow B^{\prime}+\gamma \cdot B$ (as well as $B^{\prime}$ ) is new vector or scalar bosons. In this double-decay scenario, there are three vertices with new physics interactions: $N$ production, $N$ decay, and $B$ decay.

If $B$ decays fast, so that the decay length is smaller than (or comparable to) the size of the detector, effectively the picture of transitions will be similar to that of the $U_{N} D_{\xi}$ scenario. Correspondingly, time evolution, signatures, and the most relevant experiments will be similar. The only difference is that in the $\xi=e^{+} e^{-}$case the invariant mass of the pair is fixed by the mass of $B: W_{e e}=m_{B}$. In what follows, we will consider the case $\xi=e^{+} e^{-}$, that is, the $U_{N} D_{B} D_{e e}$ scenario with fast $B$ decay.

If $m_{B}>W_{c}^{\mathrm{ND}}=5 \mathrm{MeV}, \mathrm{ND} 280$ can provide a direct test of this scenario and, therefore, give the most stringent bound. The dependence of number of events, $N_{e e-2 s h}^{\mathrm{ND}}$, on $c \tau^{0}$ is shown in the left panel in Fig. 12. It has the typical dependence with two flat asymptotics and a bump at about $0.1 \mathrm{~m}$ due to $N$ production in the outer P0D detector. [This is similar to Figs. 9 (left) and 11 (right).] For our computations, we use the partially coherent cross section. The signature factor enhancement is absent for $m_{B} \leq$ $W_{c}^{\mathrm{MB}}=30 \mathrm{MeV}$; MiniBooNE does not resolve the pair and, therefore, $f_{e e-1 s h}^{\mathrm{MB}} \approx 1$. On the other hand, for $m_{B} \gg W_{c}^{\mathrm{ND}}=5 \mathrm{MeV}$, the ND280 do resolve the pair, so that $f_{e e-2 s h}^{\mathrm{ND}} \approx 1$. For larger $m_{B}$, one would expect suppression of $f_{e e-1 s h}^{\mathrm{MB}}$ and, consequently, the signature factor enhancement of the number of events. Still, there is a weak dependence of number of events on $m_{N}$ due to partially coherent cross section dependence and slightly higher effective energy of ND280 than that of MiniBooNE. The reason is the same as for MINER $\nu \mathrm{A}$ test of $U_{N} D_{\gamma}$ scenario described in Sec. V B.

The experimental bounds in Eq. (88) (the same as in Fig. 9 left) disfavor this scenario at more than $1 \sigma$ C.L. in the whole applicable range of $c \tau^{0} \quad\left(\gtrsim 10^{-2} \mathrm{~m}\right)$ and for $m_{B}>10 \mathrm{MeV}$. In the region $c \tau^{0} \sim 10^{-1} \mathrm{~m}$, the exclusion of the scenario surpasses $3 \sigma$. With a further decrease of $m_{B}$ 
(approaching $W_{c}^{\mathrm{ND}}$ ), the number of events is suppressed by the signature factor. For $m_{B}<5 \mathrm{MeV}$, the ND280 bound on the two-shower events is not applicable, but one can use various indirect tests.

A useful indirect test of the $U_{N} D_{B} D_{e e}$ scenario is given by the MINER $\nu$ A bounds on $\gamma$-shower events (93), which requires $e e-\gamma$ shower misidentification. In Fig. 12 (right panel), we show predictions for the number of $\gamma$-shower events at MINER $\nu \mathrm{A}$. The dependence of $N_{e e-\gamma}^{\mathrm{MV}}$ on $c \tau^{0}$ has a typical smooth step form with the bump due to $N$ production in dirt. The bump is at a larger decay length than in other experiments, $c \tau^{0}=(0.5-3) \mathrm{m}$, due to larger distance between the detector and outer material. The purple and green regions correspond to $\mathrm{ME}$ and $\mathrm{LE}$ datasets, respectively. The strong dependence of the number of events on $m_{N}$ is due to the coherent cross section enhancement, as explained around Eq. (101). Much stronger dependence of $m_{N}$ in the right panel compared to the one in the left panel is related to higher neutrino energies at MINER $\nu \mathrm{A}$ and, therefore, weaker suppression of the cross section with increase of $m_{N}$ than at ND280 and MiniBooNE. Also for this reason, the prediction for the ME sample is higher than for the LE sample (in addition, the ME dataset comes with $\sim 3$ more POTs). The signature factor enhancement is absent here.

The predictions are at the level of $3 \sigma$ upper bounds on $\gamma$ shower events from Eqs. (93) and (94).

Our prediction is in rough agreement with Ref. [26], apart from the fact that we find stronger exclusion from the LE dataset than from the ME dataset. This could stem from the fact that we made simplifying assumptions on the experimental efficiencies, where a simulation was performed in Ref. [26].

The model [10] matches this scenario for $c \tau^{0}=$ $\mathcal{O}\left(10^{-9}\right) \mathrm{cm}, m_{B}=30 \mathrm{MeV}$, and $m_{N} \sim 0.25 \mathrm{GeV}$ and, therefore, is disfavored by MINER $\nu \mathrm{A}$.

However, such a parameter point is not excluded by ND280 because of the very small $c \tau^{0}$. Any realization of Ref. [10] with $c \tau^{0} \gtrsim 10^{-2} \mathrm{~m}$ is, however, tested at least at the level of $3 \sigma$ in accord with the left panel in Fig. 12.

The models with a scalar mediator [11,12] are not affected by the constraint from MINER $\nu \mathrm{A}$ due to the suppressed upscattering cross section. ND280 can still test this class of models through the search for $2 e$ tracks, analogously to Ref. [10]. Finally, there could be additional tests involving particle misidentification.

\section{Mixing-decay into $\nu_{e}$ scenario, $M_{N} D_{\nu} U_{e}$}

In this scenario (see Fig. 6), $N$ is produced via mixing in $\nu_{\mu}$, then $N$ decays along the beam line into $\nu_{e}, N \rightarrow \nu_{e}+B$, and, in turn, $\nu_{e}$ upscatters in a detector producing the $e$-like events in the low-energy range (if $B$ has large enough mass). In this way, an additional $\nu_{e}$ flux is generated.

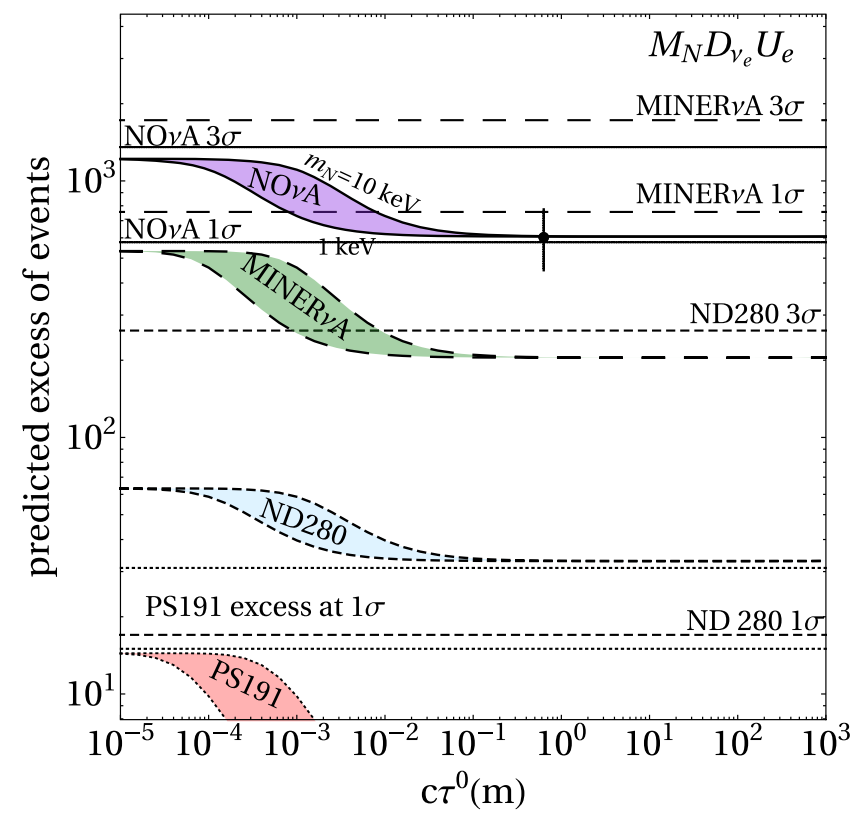

FIG. 13. Direct tests of the mixing-decay into $\nu_{e}$ scenario, $M_{N} D_{\nu}$. The number of expected events as a function of $c \tau^{0}$ for different values of $m_{N}$ (numbers at the curves in $\mathrm{keV}$ ) are shown for ND280, MINER $\nu$ A, PS191, and $\mathrm{NO} \nu \mathrm{A}$. Horizontal lines correspond to the $1 \sigma$ and $3 \sigma$ experimental upper bounds for each of these experiments. The point with the error bar indicates the uncertainty of the prediction from the MiniBooNE-observed event rate.

The direct tests of this scenario are provided by studies of the $e$-like events at ND280, MINER $\nu \mathrm{A}, \mathrm{PS} 191$, and $\mathrm{NO} \nu \mathrm{A}$ (Fig. 13). The number of events due to $M_{N} D_{\nu} U_{e}$ scenario in these experiments, $N_{e-e s h}^{i}$, has been computed using Eqs. (61) and (62). According to the analysis in Sec. III E, $N_{e-e s h}^{i}$, as functions of $c \tau^{0}$, has smooth steplike form with constant asymptotics at $c \tau^{0} \rightarrow 0$ and $c \tau^{0} \rightarrow \infty$ [see Eq. (66)] and with a transition region at

$$
c \tau^{0 i} \sim l^{i} \frac{m_{N}}{E^{i}} .
$$

Here, $l^{i}$ is the baseline. The asymptotics do not depend on $m_{N}$, and the transition region shifts with $m_{N}$, proportionally to $m_{N}$.

The limits for single $e$-shower events are given in Eqs. (90), (92), and (96). For MINER $\nu$ A, the predicted number of events is well below the $1 \sigma$ limit. The prediction for ND280 is slightly above $1 \sigma$, while, interestingly, the calculated event number for PS191 is almost consistent with the observed excess (Sec. IV). $\mathrm{NO} \nu \mathrm{A}$ disfavors this scenario at the level of $1 \sigma$ at large $c \tau^{0}$ and above $2 \sigma$ at small $c \tau^{0}$. Notice that $\mathrm{NO} \nu \mathrm{A}$ has already collected much more data with respect to the analysis presented in Ref. [42] on which our limits are based. Therefore, an updated analysis can further improve the bounds. 
The models [14,15] realize this scenario with $c \tau^{0} \sim$ $10^{-3} \mathrm{~cm}$ and $m_{M}=(1-10) \mathrm{keV}$. Therefore, with the present data the best fit point of MiniBooNE is disfavored at about $2 \sigma$.

\section{SUMMARY AND CONCLUSIONS}

We performed a model-independent study of the nonoscillatory explanations of the MiniBooNE excess in terms of the phenomenological scenarios. Here, the scenarios are series of transitions and processes which connect the initial interactions of the accelerated protons with a target and the appearance of single-shower (e-like) events in the MiniBooNE detector. The processes include the production of new particles, their propagation, and decays, as well as interactions with a medium. We parametrized scenarios by masses and decay rates of new particles as well as by cross sections.

We carried out a systematic search of the simplest scenarios which can be classified by the number of new interaction points (vertices). We have found two scenarios with two vertices, four scenarios with three vertices, etc. More possibilities are related to the nature of new propagating particles (fermions or bosons) as well as to the type of particle(s) in the final state which produce single-shower events in MiniBooNE. We show that these scenarios are reduced to a few qualitatively different configurations.

For these configurations, general formulas have been derived for the numbers of events due to new physics. Dependence of these numbers of events on parameters of the scenarios was considered. In particular, we find three qualitatively different dependences on the decay length $c \tau^{0}$ : (i) flat dependence with upturn at small $c \tau^{0}$ (scenarios with mixing), (ii) smoothed steplike dependence (scenarios with upscattering in detector), and (iii) bump followed by constant asymptotics at large $c \tau^{0}$ (scenarios with upscattering in dirt). In a sense, we developed the effective theory of new physics at low-energy accelerator experiments.

We described tests of the scenarios employing neutrino experiments which have setups similar to MiniBooNE: experiments at near detectors of $\mathrm{NO} \nu \mathrm{A}$ and T2K ND280 as well as at PS191 and MINER $\nu \mathrm{A}$. While reproducing the MiniBooNE excess, the scenarios lead to additional events in these experiments. In other words, scenarios allow one to directly connect the observed MiniBooNE excess of events to expected excesses in other experiments. In practice, we normalize the expected number of events in a given experiment to the MiniBooNE excess, and in this way various parameters and uncertainties cancel out.

For each experiment under consideration, we obtained the upper bounds on possible numbers of events due to new physics. We confronted these bounds with expected number of events related to MiniBooNE excess.

We find that, in spite of the large strength of MiniBooNE (mass and POT), other experiments produce substantial bounds due to better particle ID, higher neutrino energies, specific dependence of the cross section on the mass of a produced particle, etc. In particular, we find the signature factor enhancement and the coherent cross section enhancement.

Each of the studied scenarios can be tested, with a certain part of parameter space excluded, using available neutrino data. In particular, $U_{N} D_{e e}$ and $U_{N} D_{B} U_{e e}$ scenarios are restricted by the $2 e$-track data from ND280, while $U_{N} D_{\gamma}$ is excluded by data on isolated photons from MINER $\nu$ A. As far as the MD scenarios with $m_{N}<10 \mathrm{MeV}$ are concerned, they are disfavored by the ND280 $2 e$-tracks (higher masses are already excluded by MiniBooNE timing data). According to the $M_{N} D_{\nu} U_{e}$ scenario, a significant excess of events should already be seen at $\mathrm{NO} \nu \mathrm{A}$ with the present tension at the $2-3 \sigma$ level.

Concerning specific models, we find that Ref. [5] is disfavored at more than $3 \sigma$ by MINER $\nu \mathrm{A}$ data. The model in Ref. [6] has already been excluded by the MiniBooNE timing analysis; hence, we studied the realization of such a model with smaller right-handed neutrino masses $[\mathcal{O}(10) \mathrm{MeV}]$; both ND280 and MINER $\nu \mathrm{A}$ can exclude the models at the $3 \sigma$ level. The proposals in Refs. [7,9] with the benchmark point $c \tau^{0}>10 \mathrm{~cm}$ are disfavored by the $2 e$ track searches at ND280. The same model with prompt RH neutrino decay [8] is unconstrained by ND280, because the tracks inside the FGD detector are vetoed. In a similar way, proposals in Refs. [11-13,18] also evade limits from ND280 and are also unconstrained by MINER $\nu$ A data. In contrast, the latter disfavors the model in Ref. [10]. We found that in a class of models with light scalars and sterile neutrinos [14-17] about $O\left(10^{3}\right)$ additional events are expected in the $\mathrm{NO} \nu \mathrm{A}$ detector, which corresponds to the $2 \sigma$ upper limit. New experimental results will further strengthen these bounds. Hence, using $3 \sigma$ as a criterion, we found that benchmark points in Refs. [5-7,9,10] are ruled out and Refs. [14-17] are in tension, while Refs. [8,1113,18] are still allowed.

The scenarios and bounds we have elaborated can be useful for construction of new models which are aimed at explanations of the MiniBooNE excess. Our consideration can also be applied to new physics search without reference (connection) to the MiniBooNE excess. In this case, the expected number of events at MiniBooNE can be smaller or much smaller than the observed excess. Hence, given our general approach, this work can also be regarded as the effective theory of new physics at accelerator-based neutrino experiments, being relevant for future projects such as DUNE.

\section{ACKNOWLEDGMENTS}

O. F. thanks Bill Louis for fruitful email correspondence. V. B. thanks Sumit Ghosh and Joachim Kopp for useful discussions. Additionally, we thank Laura Fields, Matheus Hostert, and the authors of Ref. [15] for a fruitful email exchange. 
[1] A. A. Aguilar-Arevalo et al. (MiniBooNE Collaboration), Significant Excess of ElectronLike Events in the MiniBooNE Short-Baseline Neutrino Experiment, Phys. Rev. Lett. 121, 221801 (2018).

[2] A. Aguilar-Arevalo et al. (MiniBooNE Collaboration), Updated MiniBooNE neutrino oscillation results with increased data and new background studies, Phys. Rev. D 103, 052002 (2021).

[3] J. Asaadi, E. Church, R. Guenette, B. J. P. Jones, and A. M. Szelc, New light Higgs boson and short-baseline neutrino anomalies, Phys. Rev. D 97, 075021 (2018).

[4] M. Dentler, A. Hernández-Cabezudo, J. Kopp, P. A. Machado, M. Maltoni, I. Martinez-Soler, and T. Schwetz, Updated Global analysis of neutrino oscillations in the presence of eV-scale sterile neutrinos, J. High Energy Phys. 08 (2018) 010.

[5] S. N. Gninenko, The MiniBooNE Anomaly and Heavy Neutrino Decay, Phys. Rev. Lett. 103, 241802 (2009).

[6] O. Fischer, A. Hernández-Cabezudo, and T. Schwetz, Explaining the MiniBooNE excess by a decaying sterile neutrino with mass in the $250 \mathrm{MeV}$ range, Phys. Rev. D 101, 075045 (2020).

[7] P. Ballett, S. Pascoli, and M. Ross-Lonergan, U(1)' mediated decays of heavy sterile neutrinos in MiniBooNE, Phys. Rev. D 99, 071701 (2019).

[8] A. Abdullahi, M. Hostert, and S. Pascoli, A sark seesaw solution to low energy anomalies: MiniBooNE, the muon $(g-2)$, and BABAR, arXiv:2007.11813.

[9] P. Ballett, M. Hostert, and S. Pascoli, Dark neutrinos and a three portal connection to the standard model, Phys. Rev. D 101, 115025 (2020).

[10] E. Bertuzzo, S. Jana, P. A. N. Machado, and R. Zukanovich Funchal, Dark Neutrino Portal to Explain MiniBooNE Excess, Phys. Rev. Lett. 121, 241801 (2018).

[11] A. Datta, S. Kamali, and D. Marfatia, Dark sector origin of the KOTO and MiniBooNE anomalies, Phys. Lett. B 807, 135579 (2020).

[12] B. Dutta, S. Ghosh, and T. Li, Explaining $(g-2)_{\mu, e}$, KOTO anomaly and MiniBooNE excess in an extended Higgs model with sterile neutrinos, Phys. Rev. D 102, 055017 (2020).

[13] W. Abdallah, R. Gandhi, and S. Roy, A two-Higgs doublet solution to the LSND, MiniBooNE and muon $g-2$ anomalies, arXiv:2010.06159.

[14] A. de Gouvâ, O. L. G. Peres, S. Prakash, and G. V. Stenico, On the decaying-sterile neutrino solution to the electron (anti)neutrino appearance anomalies, J. High Energy Phys. 07 (2020) 141.

[15] M. Dentler, I. Esteban, J. Kopp, and P. Machado, Decaying sterile neutrinos and the short baseline oscillation anomalies, Phys. Rev. D 101, 115013 (2020).

[16] Y. Bai, R. Lu, S. Lu, J. Salvado, and B. A. Stefanek, Three twin neutrinos: Evidence from LSND and MiniBooNE, Phys. Rev. D 93, 073004 (2016).

[17] S. Palomares-Ruiz, S. Pascoli, and T. Schwetz, Explaining LSND by a decaying sterile neutrino, J. High Energy Phys. 09 (2005) 048.

[18] W. Abdallah, R. Gandhi, and S. Roy, Understanding the MiniBooNE and the muon $g-2$ anomalies with a light $Z^{\prime}$ and a second Higgs doublet, J. High Energy Phys. 12 (2020) 188.

[19] T. Katori, V. Kostelecky, and R. Tayloe, Global threeparameter model for neutrino oscillations using Lorentz violation, Phys. Rev. D 74, 105009 (2006).

[20] Y. Farzan, T. Schwetz, and A. Y. Smirnov, Reconciling results of LSND, MiniBooNE and other experiments with soft decoherence, J. High Energy Phys. 07 (2008) 067.

[21] A. Aguilar-Arevalo et al. (MiniBooNE DM Collaboration), Dark matter search in nucleon, pion, and electron channels from a proton beam dump with MiniBooNE, Phys. Rev. D 98, 112004 (2018).

[22] J. R. Jordan, Y. Kahn, G. Krnjaic, M. Moschella, and J. Spitz, Severe Constraints on New Physics Explanations of the MiniBooNE Excess, Phys. Rev. Lett. 122, 081801 (2019).

[23] K. Abe et al. (T2K Collaboration), Measurement of the charged-current electron (anti-)neutrino inclusive crosssections at the T2K off-axis near detector ND280, J. High Energy Phys. 10 (2020) 114.

[24] Bill Louis (private communication).

[25] K. Abe et al. (T2K Collaboration), Search for neutralcurrent induced single photon production at the ND280 near detector in T2K, J. Phys. G 46, 08LT01 (2019).

[26] C. A. Argelles, M. Hostert, and Y.-D. Tsai, Testing New Physics Explanations of MiniBooNE Anomaly at Neutrino Scattering Experiments, Phys. Rev. Lett. 123, 261801 (2019).

[27] D. Kim, P. A. Machado, J.-C. Park, and S. Shin, Optimizing energetic light dark matter searches in dark matter and neutrino experiments, J. High Energy Phys. 07 (2020) 057.

[28] J. A. Formaggio and G. P. Zeller, From eV to EeV: Neutrino cross sections across energy scales, Rev. Mod. Phys. 84, 1307 (2012).

[29] A. A. Aguilar-Arevalo et al. (MiniBooNE Collaboration), The neutrino flux prediction at MiniBooNE, Phys. Rev. D 79, 072002 (2009).

[30] https://www-boone.fnal.gov/for_physicists/data_release/nue_ nuebar_2012/efficiency/MB_nu_nubar_combined_release .html.

[31] K. Abe et al. (T2K Collaboration), The T2K experiment, Nucl. Instrum. Methods Phys. Res., Sect. A 659, 106 (2011).

[32] Y. Kudenko (T2K Collaboration), The near neutrino detector for the T2K experiment, Nucl. Instrum. Methods Phys. Res., Sect. A 598, 289 (2009).

[33] S. Assylbekov et al., The T2K ND280 off-axis pi-zero detector, Nucl. Instrum. Methods Phys. Res., Sect. A 686, 48 (2012).

[34] P. Amaudruz et al. (T2K ND280 FGD Collaboration), The T2K fine-grained detectors, Nucl. Instrum. Methods Phys. Res., Sect. A 696, 1 (2012).

[35] K. Abe et al. (T2K Collaboration), T2K neutrino flux prediction, Phys. Rev. D 87, 012001 (2013); Phys. Rev. D 87, 019902(A) (2013).

[36] K. Abe et al. (T2K Collaboration), Search for heavy neutrinos with the T2K near detector ND280, Phys. Rev. D 100, 052006 (2019).

[37] J. Wolcott et al. (MINERvA Collaboration), Measurement of Electron Neutrino Quasielastic and Quasielasticlike 
Scattering on Hydrocarbon at $\left\langle E_{\nu}\right\rangle=3.6 \mathrm{GeV}$, Phys. Rev. Lett. 116, 081802 (2016).

[38] J. Park et al. (MINERvA Collaboration), Measurement of neutrino flux from neutrino-electron elastic scattering, Phys. Rev. D 93, 112007 (2016).

[39] E. Valencia et al. (MINERvA Collaboration), Constraint of the MINER $\nu \mathrm{A}$ medium energy neutrino flux using neutrinoelectron elastic scattering, Phys. Rev. D 100, 092001 (2019).

[40] G. Bernardi et al., Further limits on heavy neutrino couplings, Phys. Lett. B 203, 332 (1988).

[41] G. Bernardi, G. Carugno, J. Chauveau, F. Di Carlo, M. Dris, J. Dumarchez, M. Ferro-Luzzi, J. M. Lvy, D. Lukas,
J. M. Perreau, Y. Pons, A. M. Touchard, and F. Vannucci, Anomalous electron production observed in the CERN PS neutrino beam, Phys. Lett. B 181, 173 (1986).

[42] P. Adamson et al. (NOvA Collaboration), First Measurement of Electron Neutrino Appearance in NOvA, Phys. Rev. Lett. 116, 151806 (2016).

[43] C. T. Kullenberg et al. (NOMAD Collaboration), A search for single photon events in neutrino interactions, Phys. Lett. B 706, 268 (2012).

[44] Alexander Izmaylov (private communication).

[45] P. Coloma, Icecube/DeepCore tests for novel explanations of the MiniBooNE anomaly, Eur. Phys. J. C 79, 748 (2019). 